\title{
Computation of the Nash Equilibrium Selected by the Tracing Procedure in N-Person Games ${ }^{1}$
}

\author{
P. Jean-Jacques Herings and Antoon van den Elzen \\ Department of Economics, University of Maastricht, P.O. Box 616, 6200 MD, \\ Maastricht, The Netherlands \\ E-mail: P.Herings@algec.unimaas.nl
}

Received October 20, 1999

\begin{abstract}
The heart of the equilibrium selection theory of Harsanyi and Selten (1988, A General Theory of Equilibrium Selection in Games, Cambridge, MA: MIT Press) is given by the tracing procedure, a mathematical construction that adjusts arbitrary prior beliefs into equilibrium beliefs. Although the term "procedure" suggests a numerical approach, the tracing procedure itself is a nonconstructive method. In this paper we propose a homotopy algorithm that generates a path of strategies. By using lexicographic pivoting techniques, it can be shown that for the entire class of noncooperative $N$-person games, the path converges to an approximate Nash equilibrium, even when the starting point or the game is degenerate. The outcome of the algorithm is shown to be arbitrarily close to the equilibrium beliefs proposed by the tracing procedure. Therefore, the algorithm does not compute just any Nash equilibrium, but one with a sound game-theoretic underpinning. Like other homotopy algorithms, it is easily implemented on a computer. Journal of Economic Literature Classification Numbers: C63, C72. ๑ 2002 Elsevier Science

Key Words: computation of equilibria; noncooperative game theory; tracing procedure.
\end{abstract}

\section{INTRODUCTION}

In years past, economics has benefited greatly from the introduction of game-theoretic tools. A further use of game theory is hampered by at least two factors, however. First, there may be many solutions to a game. This view is expressed in van Damme (1999): "In the last two decades, game theoretic methods have become more and more important in economics and the other social sciences. Many scientific papers in these areas have

\footnotetext{
${ }^{1}$ The authors thank Dries Vermeulen and an anonymous referee for helpful comments and suggestions. The research of Jean-Jacques Herings has been made possible by a fellowship of the Royal Netherlands Academy of Arts and Sciences.
} 
the following basic structure: A problem is modeled as a game, the game is analyzed by computing its equilibria, and the properties of the latter are translated back into insights relevant to the original problem...It has been found that the tools may not be powerful enough...For example, many models admit a vast multiplicity of equilibrium outcomes so that the predictive power of game theoretic analysis is limited. To increase understanding, it may, hence, be necessary to perfect the tools." A way out of this dilemma has been suggested by the equilibrium selection theory as described in Harsanyi and Selten (1988). The main ingredient of this equilibrium selection theory is the linear tracing procedure. The linear tracing procedure plays also an important role in making risk-dominance comparisons of Nash equilibria (see Harsanyi and Selten, 1988). The concept of risk dominance is frequently used in evolutionary game theory.

A second problem is that it is usually far from obvious to derive any solution for a given game. Moreover, considering the remarks of van Damme (1999), to find just a solution is not good enough. What is really needed is a solution that has a good game-theoretic underpinning-for instance, the solution provided by the linear tracing procedure. In evolutionary game theory, attention is often focused on two-player games where each player has two strategies. An important reason to restrict oneself to these games is that it is very hard to determine the risk-dominance relationships for Nash equilibria in more complicated games. To do this, one needs to apply the linear tracing procedure several times to these games, which generally is not possible without a numerical algorithm.

This paper presents an algorithm that computates the Nash equilibrium selected by the linear tracing procedure for $N$-person noncooperative games in normal form. The linear tracing procedure operates as follows. The players start with identical initial beliefs concerning the play of the other players, so that the beliefs concerning the play of a certain player are equal among all players. First, the players optimize by playing best replies against these initial beliefs. Next, they observe that their beliefs are not met, and they subsequently update their beliefs and react optimally on them. This updating of beliefs continues until equilibrium beliefs for the game are found.

Mathematically, the linear tracing procedure can be modeled as tracing a path of 0's related to a homotopy. The homotopy transforms the initial problem of playing against the prior beliefs into the equilibrium problem of the game. It has been shown by Harsanyi (1975) that for a generic game, the linear tracing procedure yields a path of points and selects a unique outcome. In this case the linear tracing procedure is said to be well defined. For any game, the linear tracing procedure yields a set of strategies that connects a best reply to the prior beliefs to at least one Nash equilibrium of the game. If one wants to select a unique Nash equilibrium even in 
the exceptional case where multiple Nash equilibria are connected to the prior beliefs, one possibility is to resort to the logarithmic tracing procedure. Whenever the linear tracing procedure is well defined, the logarithmic tracing procedure will select the same outcome (see Schanuel et al., 1991).

The case with $N=2$ players corresponds to the class of bimatrix games. The first algorithm for solving for a Nash equilibrium of these games has been given by Lemke and Howson (1964). A drawback of this method is that it must be started at a vertex of the strategy space. Implementational details of a modified Lemke-Howson algorithm are discussed in Krohn et al. (1991). More flexible than the Lemke-Howson method is the one proposed by van den Elzen and Talman (1991). This method can be started from any strategy vector in the strategy space. In van den Elzen and Talman (1999), it is shown that their algorithm generates a nonlinear transformation of the path of beliefs corresponding to the path as generated by the linear tracing procedure. This nonlinear transformation guarantees that the path generated by the van den Elzen and Talman (1999) algorithm is piecewise linear for bimatrix games, and thus is easily implemented on a computer. This linear approach can also be extended to polymatrix games (see van den Elzen, 1996), but not beyond.

The general case with $N>2$ is considerably more difficult. For this case, the Nash equilibrium equations are nonlinear and in general impossible to solve analytically. Therefore, one must use a numerical approach. The first procedures for finding an equilibrium for $N$-person games were developed by Rosenmüller (1971) and Wilson (1971). Both methods can be seen as different generalizations of the procedure of Lemke and Howson for twoplayer games. Although these methods are not directly suitable for computational purposes, because they merely prove the existence of a nonlinear path leading to an equilibrium, their seminal work was a very important step toward an implementable algorithm as developed by Garcia et al. (1973). A more efficient algorithm was developed in van der Laan and Talman (1982) that operates directly on the strategy space and that can be restarted at any point in the strategy space. The problem with these algorithms is that they just calculate an approximation of a sample Nash equilibrium and do not address the game-theoretic properties of the equilibrium that is found.

In this paper we present a method that follows the set of strategies implicitly defined by the linear tracing procedure arbitrarily close. In this way we make the linear tracing procedure operational for $N$-player games. The entire path of strategy vectors generated has a game-theoretic interpretation (see Harsanyi and Selten, 1988). Unlike the algorithm for the two-player case of van den Elzen and Talman (1999), our algorithm works directly on the same strategy space and with the same payoff function as used in the linear tracing procedure. The entire space is given by $[0,1] \times S$, with $[0,1]$ the interval from which the homotopy parameter, $t$, is chosen and 
$S$ the Cartesian product of the $N$ individual strategy sets. By triangulating the set $[0,1] \times S$, we replace the original problem by a piecewise linear one. The algorithm belongs to the class of homotopy algorithms first developed in Eaves (1972). The algorithm is constructed in such a way that any triangulation can be used. Of course, in practice one would like to take a triangulation that can be implemented easily on a computer.

A novelty of the algorithm is that the starting point is endogenously determined by the game's payoffs. We show that the algorithm selects in every game a unique vertex of the strategy space that is related to Nash equilibrium play against the prior. (This latter property may be satisfied for many vertices.) The starting point is chosen such that the algorithm will not generate any other simplices in $\{0\} \times S$. We prove that we can approximate the entire path of a well-defined linear tracing procedure arbitrarily close by taking simplices with small enough mesh size. If the linear tracing procedure is not well defined, then we will still stay arbitrarily close to the set of strategies generated by it, and will compute an approximation of a Nash equilibrium in this set. These features are also new, since unlike for other simplicial algorithms, here we have to consider the convergence of a sequence of paths and not only the convergence of a sequence of endpoints. Otherwise we cannot guarantee that the Nash equilibrium selected by the linear tracing procedure is also selected by the algorithm. These problems do not occur for the two-player case, since in that case it is possible to generate the path of the linear tracing procedure exactly. To handle degeneracies, we use lexicographic pivoting techniques. Degeneracies are important in game theory and are often the rule rather than the exception.

The paper is organized as follows. In Section 2 we give some notation and the definition of the linear tracing procedure. In Section 3 we treat some preliminaries for piecewise linear methods, and in Section 4 we explain the algorithm, which is illustrated in Section 5 by means of an example. In Section 6 we prove the convergence of the algorithm for any game. This implies that for every game, we can compute an approximate Nash equilibrium. In Section 7 we show that if the mesh size of the triangulation used goes to 0 , then the paths generated by the algorithm converge to the path of the linear tracing procedure and the approximate Nash equilibria converge to the Nash equilibrium selected by the linear tracing procedure.

\section{THE LINEAR TRACING PROCEDURE}

For $m \in \mathbb{N}$, let $\mathbb{R}_{+}^{m}$ be the nonnegative orthant of the $m$-dimensional Euclidean space and let $S^{m}$ denote the $(m-1)$-dimensional unit simplex in $\mathbb{R}^{m}$, i.e. $S^{m}=\left\{x \in \mathbb{R}_{+}^{m} \mid \sum_{j=1}^{m} x_{j}=1\right\}$. For $j=1, \ldots, m$, we denote by $e^{m}(j)$ the $j$ th $m$-dimensional unit vector. Furthermore, $0^{m}$ and 
$1^{m}$ denote the $m$-dimensional vectors of all 0 's and all 1's, respectively. The $m$-dimensional unit matrix is denoted by $I^{m}$. The notation "co" is used to denote the convex hull of a set.

An $N$-person noncooperative game in normal form is a tuple $\Gamma=$ $\left(\Phi_{1}, \ldots, \Phi_{N}, R_{1}, \ldots, R_{N}\right)$, with $\Phi_{i}$ and $R_{i}: \prod_{i \in \mathcal{N}} \Phi_{i} \rightarrow \mathbb{R}$ is the set of pure strategies and the payoff function of player $i$, and $\mathcal{N}=\{1, \ldots, N\}$ the set of players. Player $i$ has $M_{i}$ pure strategies. The total number of pure strategies is given by $M=\sum_{i \in \mathcal{N}} M_{i}$. We number the pure strategies of player $i$. For $k=1, \ldots, M_{i}$, pure strategy $k$ of player $i$ is denoted by $(i, k)$. The union of the pure strategies over all players is denoted by $\Phi_{*}=\cup_{i \in \mathcal{N}} \Phi_{i}$. The set of pure strategy combinations is given by $\Phi=\prod_{i \in \mathcal{N}} \Phi_{i}$.

A mixed strategy of player $i$ is a probability distribution on $\Phi_{i}$. Since the strategies are numbered, we can identify the set of all probability distributions on $\Phi_{i}$ with $S^{M_{i}}$, where for $s_{i} \in S^{M_{i}}$ the probability assigned to pure strategy $(i, k)$ is given by $s_{i k}$. With a slight abuse of notation, we also denote by $(i, k)$ the mixed strategy $s_{i}$ that assigns weight 1 to pure strategy $(i, k)$, when no confusion is possible. The strategy space of the game is equal to $S=\prod_{i \in \mathcal{N}} S^{M_{i}}$. Observe that the dimension of $S$ equals $M-N$. Given a mixed strategy combination $s \in S$ and a strategy $\bar{s}_{i} \in S^{M_{i}}$, we denote by $s \backslash \bar{s}_{i}$ the mixed strategy combination that results from replacing $s_{i}$ by $\bar{s}_{i}$. If a mixed strategy combination $s$ is played, then the probability $s(\phi)$ that the pure strategy combination $\phi=\left(\left(1, k_{1}\right), \ldots,\left(N, k_{N}\right)\right)$ occurs is given by

$$
s(\phi)=\prod_{i \in \mathcal{N}} s_{i k_{i}},
$$

and the expected payoff of player $i$ is given by

$$
R_{i}(s)=\sum_{\phi \in \Phi} s(\phi) R_{i}(\phi) .
$$

A mixed strategy combination $s$ is said to be a Nash equilibrium of a game $\Gamma$ if it is a best reply against itself. The set of Nash equilibria of a game $\Gamma$ is denoted by $\mathrm{NE}(\Gamma)$.

For the remainder of the paper, an $N$-person noncooperative game $\Gamma$ is assumed to be given. In the description of the linear tracing procedure, a subjective probability distribution $p \in S$, called the prior, is given. The prior describes the initial beliefs of all players about the strategies played by the other players. So it is assumed that all players have the same initial beliefs. The determination of the prior is one of the aspects of the equilibrium selection theory of Harsanyi and Selten (1988). Throughout the remainder of the paper, a prior $p$ is assumed to be given.

For every $t \in[0,1]$, the linear tracing procedure generates a Nash equilibrium of a game $\Gamma^{t}=\left(\Phi_{1}, \ldots, \Phi_{N}, R_{1}^{t}, \ldots, R_{N}^{t}\right)$, where the payoff function $R_{i}^{t}: \Phi \rightarrow \mathbb{R}$ of player $i$ is defined by

$$
R_{i}^{t}(\phi)=t R_{i}(\phi)+(1-t) R_{i}\left(p \backslash \phi_{i}\right) .
$$


Together, all of the Nash equilibria generated by the linear tracing procedure yields a set of strategies (usually a path of strategies) linking a Nash equilibrium of $\Gamma^{0}$ to a Nash equilibrium of $\Gamma^{1}$. In a Nash equilibrium $s^{t}$ of the game $\Gamma^{t}$, every player $i$ plays a best reply in the game $\Gamma$ against the probability distribution $t[s]+(1-t)[p]$ on $\Phi$, with $[s]$ and $[p]$ the probability distributions on $\Phi$ generated by $s$ and $p$, respectively. The probability distribution $t[s]+(1-t)[p]$ generally does not belong to $S$, since this probability distribution may be correlated. The interpretation of the linear tracing procedure is that players gradually adjust their beliefs about the behavior of the other players, giving less and less weight to the initial beliefs, the prior. In the game $\Gamma^{0}$, for instance, there is no strategic interaction, and players simply choose a best reply to the prior. In the game $\Gamma^{1}$, there is no longer a role for the prior, and players choose a best reply against the choices of their opponents. (For more details on the tracing procedure, see Harsanyi and Selten, 1988.)

The marginal payoff function $G:[0,1] \times S \rightarrow \mathbb{R}^{M}$ is defined by

$$
G_{i k}(t, s)=t R_{i}(s \backslash(i, k))+(1-t) R_{i}(p \backslash(i, k)), \quad(i, k) \in \Phi_{*} .
$$

$G_{i k}(t, s)$ is the payoff to player $i$ when playing pure strategy $(i, k)$ against the mixed strategy combination $s$ in game $\Gamma^{t}$ or, alternatively, the payoff to player $i$ when playing pure strategy $(i, k)$ against the probability distribution $t[s]+(1-t)[p]$ on $\Phi$ in the game $\Gamma$. It is standard to characterize a Nash equilibrium of the game $\Gamma^{t}$ in terms of $G(t, \cdot)$. Indeed, $s$ is a Nash equilibrium of $\Gamma^{t}$ if and only if for all $(i, k) \in \Phi_{*}, s_{i k}>0$ implies $G_{i k}(t, s)=\max _{(i, l) \in \Phi_{i}} G_{i l}(t, s)$. Therefore, it makes sense to define for $\epsilon \geq 0$ an $\epsilon$-Nash equilibrium of $\Gamma^{t}$ as follows.

Definition 2.1. Let $(\Gamma, p)$ be given. For $\epsilon \geq 0$, a mixed strategy combination $s \in S$ is called an $\epsilon$-Nash equilibrium of $\Gamma^{t}$ if $s_{i k}>0$ implies $G_{i k}(t, s) \geq \max _{(i, l) \in \Phi_{i}} G_{i l}(t, s)-\epsilon$.

A player can increase his payoff in an $\epsilon$-Nash equilibrium of $\Gamma^{t}$ at most by $\epsilon$ by choosing a best reply. The set of all Nash equilibria related to the games $\Gamma^{t}, t \in[0,1]$, is denoted by

$$
\mathscr{L}=\left\{(t, s) \in[0,1] \times S \mid s \in \mathrm{NE}\left(\Gamma^{t}\right)\right\} .
$$

The linear tracing procedure is said to be feasible if there exists a path in $\mathscr{L}$ connecting a best reply against the prior to a Nash equilibrium of the game $\Gamma$; i.e., there exists a continuous function $\gamma:[0,1] \rightarrow \mathscr{L}$ such that $\gamma(0) \in \mathscr{L} \cap(\{0\} \times S)$ and $\gamma(1) \in \mathscr{L} \cap(\{1\} \times S)$. It is shown in Schanuel et al. (1991) that the linear tracing procedure is always feasible. However, there may be many trajectories $\gamma([0,1])$ that link a Nash equilibrium of $\Gamma^{0}$ to a Nash equilibrium of $\Gamma^{1}$. If this trajectory is unique, then the linear 
tracing procedure is said to be well defined. If the linear tracing procedure is well defined, then it selects a unique Nash equilibrium of the game $\Gamma$. It is shown by Harsanyi (1975) that, given a prior, the linear tracing procedure is well defined for almost all $N$-person noncooperative games.

It is obvious that the set $\mathscr{L}^{0}=\mathscr{L} \cap(\{0\} \times S)$ is connected. In fact, because of the linearity of $G(0, \cdot)$, it holds that $\mathscr{L}^{0}$ is a polytope, the set of best replies against $p$. We denote the component (i.e., a maximally connected subset) of $\mathscr{L}$ that contains $\mathscr{L}^{0}$ by $\mathscr{L}^{c}$. Since $\mathscr{L}$ is a semialgebraic set (see Schanuel et al., 1991), it holds that $\mathscr{L}^{\mathrm{c}}$ is pathconnected. The linear tracing procedure is feasible if and only if $\mathscr{L}^{\mathrm{c}} \cap(\{1\} \times S) \neq \varnothing$. An implication of the well definedness of the linear tracing procedure is that both $\mathscr{L}^{\mathrm{c}} \cap$ $(\{0\} \times S)$ and $\mathscr{L}^{\mathrm{c}} \cap(\{1\} \times S)$ consist of a single element.

If the number of players is equal to two, then it is possible to compute the set $\mathscr{L}^{c}$ exactly. However, even in that case there is a need for a systematic approach as given in van den Elzen and Talman (1999). If the number of players is greater than or equal to three, then computing the set $\mathscr{L}^{\mathrm{c}}$ corresponds to solving a higher-order polynomial, which cannot be done in general. In that case there is a need not only for a systematic approach, but also for a numerical algorithm.

\section{A PIECEWISE LINEAR APPROACH}

The basic idea for the algorithm is relatively simple. We approximate the marginal payoff function $G$ as given in Eq. (1) by a function that is piecewise linear on $[0,1] \times S$. Then we can solve for the set $\mathscr{L}^{c}$ corresponding to this piecewise linear approximation.

Let us discuss some preliminaries related to piecewise linear approximations. For given $m \in \mathbb{N}$, an $m$-dimensional simplex or $m$-simplex $\sigma$ in $\mathbb{R}^{n}$ is defined as the convex hull of $m+1$ affinely independent points $x^{1}, \ldots, x^{m+1}$ of $\mathbb{R}^{n}$. We usually write $\sigma=\sigma\left(x^{1}, \ldots, x^{m+1}\right)$ and call $x^{1}, \ldots, x^{m+1}$ the vertices of $\sigma$. An $(m-1)$-simplex that is, the convex hull of $m$ vertices of $\sigma\left(x^{1}, \ldots, x^{m+1}\right)$, is said to be a facet of $\sigma$. The facet $\tau\left(x^{1}, \ldots, x^{j-1}, x^{j+1}, \ldots, x^{m+1}\right)$ is called the facet of $\sigma$ opposite to the vertex $x^{j}$. For a nonnegative integer $m^{\prime}$ less than or equal to $m$, an $m^{\prime}$-simplex that is the convex hull of $m^{\prime}+1$ vertices of $\sigma$ is said to be an $m^{\prime}$-face or face of $\sigma$.

A finite collection $\Sigma$ of $m$-simplices is a triangulation of an $m$-dimensional convex subset $T$ of $\mathbb{R}^{n}$ if (1) $T$ is the union of all simplices in $\Sigma$ and (2) the intersection of any two simplices in $\Sigma$ is either empty or a common face of both. If $\Sigma$ is a triangulation of $T$, and a facet $\tau$ of $\sigma^{1} \in \Sigma$ is a subset of the relative boundary of $T$, then there is no $\sigma^{2} \in \Sigma$ such that $\sigma^{2} \neq \sigma^{1}$ and $\tau$ is a facet of $\sigma^{2}$. If $\tau$ is not a subset of the relative boundary of $T$, 


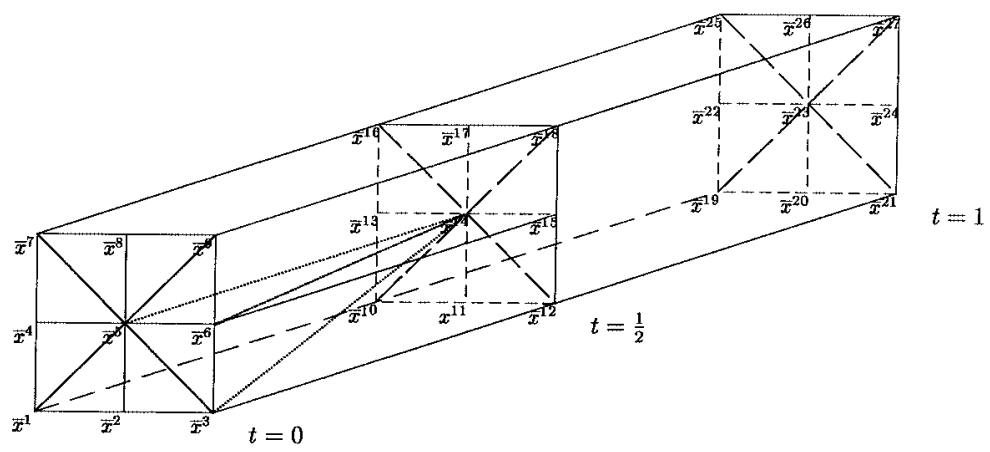

FIG. 1. A triangulation of $[0,1] \times S$. The vertex $\bar{x}^{1}=(0,(1,0),(1,0))$ refers to the strategy vector at which both players play their first pure strategy. Similarly, $\bar{x}^{3}, \bar{x}^{7}$, and $\bar{X}^{9}$ correspond to $(0,(0,1),(1,0)),(0,(1,0),(0,1))$, and $(0,(0,1),(0,1))$, respectively.

then there is exactly one $\sigma^{2} \in \Sigma$ such that $\sigma^{2} \neq \sigma^{1}$ and $\tau$ is also a facet of $\sigma^{2}$. The mesh size of a triangulation $\Sigma$ of $T$ is defined by $\operatorname{mesh}(\Sigma)=$ $\max \left\{\|x-y\|_{\infty} \mid x, y \in \sigma, \sigma \in \Sigma\right\}$.

It is well known that full-dimensional affine parts of the relative boundary of a set are triangulated by the facets of the simplices in a triangulation. More precisely, let $\Sigma$ be a triangulation of a convex $m$-dimensional subset $T$ of $\mathbb{R}^{n}$, and let the $(m-1)$-dimensional subset $\bar{T}$ of the relative boundary of $T$ be such that $\bar{T}$ is equal to the affine hull of $\bar{T}$ intersected with $T$. Then the collection $\bar{\Sigma}$ given by $\bar{\Sigma}=\{\tau \in \bar{T} \mid \exists \sigma \in \Sigma, \tau$ is a facet of $\sigma\}$ is a triangulation of $\bar{T}$ (see Todd, 1976a), Theorem 2.3, p. 27). For instance, the set $\{0\} \times S$ is triangulated by some facets of the simplices in a triangulation of $[0,1] \times S$.

An example of a triangulation of $[0,1] \times S$ has been introduced in Doup and Talman (1987) and uses earlier ideas of Eaves and Saigal (1972), Todd (1976b), and van der Laan and Talman (1980). It is illustrated in Figure 1 for the case where we have two players, each having two pure strategies. Although any triangulation of $[0,1] \times S$ will do for the purposes of the algorithm, this triangulation has several nice properties. First, it is easily implemented on a computer. Moreover, it has several nice symmetry properties. For example, it enables us to choose points $t^{0}, \ldots, t^{k}$ with $t^{0}=0$ and $t^{k}=1$ (a natural choice would be $t^{j}=j / k, j=0, \ldots, k$ ) such that every slice $\left\{t^{j}\right\} \times S$ is triangulated in an identical way by the facets of the simplices in the triangulation. Any simplex in this triangulation is contained in $\left[\frac{j-1}{k}, \frac{j}{k}\right] \times S$ for some $j$. So $\left[\frac{j-1}{k}, \frac{j}{k}\right] \times S$ is filled up with simplices in a consistent way. All 3 -simplices in $\operatorname{co}\left(\bar{x}^{3}, \bar{x}^{5}, \bar{x}^{6}, \bar{x}^{12}, \bar{x}^{14}, \bar{x}^{15}\right)$ are depicted in Figure 1 . The mesh size of this triangulation can be made arbitrarily small. 
TABLE I

All Full-Dimensional Simplices in $\left[0, \frac{1}{2}\right] \times S$

\begin{tabular}{llll}
\hline $\operatorname{co}\left(\left\{\bar{x}^{4}, \bar{x}^{7}, \bar{x}^{5}, \bar{x}^{14}\right\}\right)$ & $\operatorname{co}\left(\left\{\bar{x}^{8}, \bar{x}^{7}, \bar{x}^{5}, \bar{x}^{14}\right\}\right)$ & $\operatorname{co}\left(\left\{\bar{x}^{8}, \bar{x}^{9}, \bar{x}^{5}, \bar{x}^{14}\right\}\right)$ & $\operatorname{co}\left(\left\{\bar{x}^{6}, \bar{x}^{9}, \bar{x}^{5}, \bar{x}^{14}\right\}\right)$ \\
$\operatorname{co}\left(\left\{\bar{x}^{4}, \bar{x}^{7}, \bar{x}^{16}, \bar{x}^{14}\right\}\right)$ & $\operatorname{co}\left(\left\{\bar{x}^{8}, \bar{x}^{7}, \bar{x}^{16}, \bar{x}^{14}\right\}\right)$ & $\operatorname{co}\left(\left\{\bar{x}^{8}, \bar{x}^{9}, \bar{x}^{18}, \bar{x}^{14}\right\}\right)$ & $\operatorname{co}\left(\left\{\bar{x}^{6}, \bar{x}^{9}, \bar{x}^{18}, \bar{x}^{14}\right\}\right)$ \\
$\operatorname{co}\left(\left\{\bar{x}^{4}, \bar{x}^{13}, \bar{x}^{16}, \bar{x}^{14}\right\}\right)$ & $\operatorname{co}\left(\left\{\bar{x}^{8}, \bar{x}^{17}, \bar{x}^{16}, \bar{x}^{14}\right\}\right)$ & $\operatorname{co}\left(\left\{\bar{x}^{8}, \bar{x}^{17}, \bar{x}^{18}, \bar{x}^{14}\right\}\right)$ & $\operatorname{co}\left(\left\{\bar{x}^{6}, \bar{x}^{15}, \bar{x}^{18}, \bar{x}^{14}\right\}\right)$ \\
$\operatorname{co}\left(\left\{\bar{x}^{4}, \bar{x}^{1}, \bar{x}^{5}, \bar{x}^{14}\right\}\right)$ & $\operatorname{co}\left(\left\{\bar{x}^{2}, \bar{x}^{1}, \bar{x}^{5}, \bar{x}^{14}\right\}\right)$ & $\operatorname{co}\left(\left\{\bar{x}^{2}, \bar{x}^{3}, \bar{x}^{5}, \bar{x}^{14}\right\}\right)$ & $\operatorname{co}\left(\left\{\bar{x}^{6}, \bar{x}^{3}, \bar{x}^{5}, \bar{x}^{14}\right\}\right)$ \\
$\operatorname{co}\left(\left\{\bar{x}^{4}, \bar{x}^{1}, \bar{x}^{10}, \bar{x}^{14}\right\}\right)$ & $\operatorname{co}\left(\left\{\bar{x}^{2}, \bar{x}^{1}, \bar{x}^{10}, \bar{x}^{14}\right\}\right)$ & $\operatorname{co}\left(\left\{\bar{x}^{2}, \bar{x}^{3}, \bar{x}^{12}, \bar{x}^{14}\right\}\right)$ & $\operatorname{co}\left(\left\{\bar{x}^{6}, \bar{x}^{3}, \bar{x}^{12}, \bar{x}^{14}\right\}\right)$ \\
$\operatorname{co}\left(\left\{\bar{x}^{4}, \bar{x}^{13}, \bar{x}^{10}, \bar{x}^{14}\right\}\right)$ & $\operatorname{co}\left(\left\{\bar{x}^{2}, \bar{x}^{11}, \bar{x}^{10}, \bar{x}^{14}\right\}\right)$ & $\operatorname{co}\left(\left\{\bar{x}^{2}, \bar{x}^{11}, \bar{x}^{12}, \bar{x}^{14}\right\}\right)$ & $\operatorname{co}\left(\left\{\bar{x}^{6}, \bar{x}^{15}, \bar{x}^{12}, \bar{x}^{14}\right\}\right)$ \\
\hline
\end{tabular}

For later use, we give all 3-simplices in the triangulation of Figure 1 in Table I. In the table only the 3 -simplices in $\left[0, \frac{1}{2}\right] \times S$ are given, the ones in $\left[\frac{1}{2}, 1\right] \times S$ follow by means of a translation. The position in the table is related to the position of a simplex in the triangulation.

A function $\bar{G}:[0,1] \times S \rightarrow \mathbb{R}^{M}$ is called a piecewise linear approximation of the marginal payoff function $G$ with respect to $\Sigma$ if for each vertex $x^{j}$ of any $\sigma\left(x^{1}, \ldots, x^{M-N+2}\right) \in \Sigma, \bar{G}\left(x^{j}\right)=G\left(x^{j}\right)$ and $\bar{G}$ is affine on each simplex of $\Sigma$. Hence, if $x \in \sigma\left(x^{1}, \ldots, x^{M-N+2}\right)$, so that $x=\sum_{j=1}^{M-N+2} \lambda_{j} x^{j}, \lambda_{j} \geq$ $0, j=1, \ldots, M-N+2, \sum_{j=1}^{M-N+2} \lambda_{j}=1$, then $\bar{G}(x)=\sum_{j=1}^{M-N+2} \lambda_{j} \bar{G}\left(x^{j}\right)$.

Definition 3.1. Let $(\Gamma, p, \Sigma)$ be given. A mixed strategy combination $s \in S$ is called an approximate Nash equilibrium of $\Gamma^{t}$ if $s_{i k}>0$ implies $\bar{G}_{i k}(t, s)=\max _{(i, l) \in \Phi_{i}} \bar{G}_{i l}(t, s)$.

The algorithm will yield a path of approximate Nash equilibria going from an approximate Nash equilibrium of $\Gamma^{0}$ to an approximate Nash equilibrium of $\Gamma^{1}$. An approximate Nash equilibrium of $\Gamma^{t}$ is not necessarily a Nash equilibrium of $\Gamma^{t}$ (although it is possible to show that an approximate Nash equilibrium of $\Gamma^{0}$ is a Nash equilibrium of $\Gamma^{0}$ ). Nevertheless, the following result shows that it is an $\epsilon$-Nash equilibrium of $\Gamma^{t}$, with $\epsilon>0$ related to the mesh size of the triangulation.

THEOREM 3.2. Let $(\Gamma, p, \Sigma)$ be given. For every $\epsilon>0$, we can choose $\delta>0$ such that $\hat{x}, \tilde{x} \in[0,1] \times S,\|\hat{x}-\tilde{x}\|_{\infty} \leq \delta$, implies $\|G(\hat{x})-G(\tilde{x})\|_{\infty} \leq$ $\frac{1}{2} \epsilon$. If the mesh size of $\Sigma$ is less than $\delta$ and $\bar{s}$ is an approximate Nash equilibrium of $\Gamma^{\bar{t}}$, then $\bar{s}$ is an $\epsilon$-Nash equilibrium of $\Gamma^{\bar{t}}$.

Proof. Let $\epsilon>0$ be given. Since $G$ is a continuous function with domain a compact set, we can choose $\delta>0$ as in the theorem. Let the mesh size of $\Sigma$ be less than $\delta$ and let $\bar{s}$ be an approximate Nash equilibrium of $\Gamma^{\bar{t}}$. Let $\sigma \in \Sigma$ be such that $\bar{x}=(\bar{t}, \bar{s}) \in \sigma$. Then there exists $\lambda \in \mathbb{R}^{M-N+2}$ such that $\sum_{j=1}^{M-N+2} \lambda_{j}=1$ and $\bar{x}=\sum_{j=1}^{M-N+2} \lambda_{j} x^{j}$, with $x^{j}$ the vertices of $\sigma$. Then

$$
\|\bar{G}(\bar{x})-G(\bar{x})\|_{\infty}=\left\|\sum_{j=1}^{M-N+2} \lambda_{j}\left(G\left(x^{j}\right)-G(\bar{x})\right)\right\|_{\infty} \leq \frac{1}{2} \epsilon,
$$


and so, for every $(i, k) \in \Phi_{*}$,

$$
\bar{G}_{i k}(\bar{x})-\frac{1}{2} \epsilon \leq G_{i k}(\bar{x}) \leq \bar{G}_{i k}(\bar{x})+\frac{1}{2} \epsilon .
$$

Now, $\bar{s}_{i k}>0$ implies

$$
\begin{aligned}
G_{i k}(\bar{t}, \bar{s}) & =G_{i k}(\bar{x}) \geq \bar{G}_{i k}(\bar{x})-\frac{1}{2} \epsilon=\max _{(i, l) \in \Phi_{i}} \bar{G}_{i l}(\bar{x})-\frac{1}{2} \epsilon \\
& \geq \max _{(i, l) \in \Phi_{i}} G_{i l}(\bar{x})-\epsilon \\
& =\max _{(i, l) \in \Phi_{i}} G_{i l}(\bar{t}, \bar{s})-\epsilon .
\end{aligned}
$$

By Theorem 3.2, we can show that the algorithm generates a path of $\epsilon$-Nash equilibria of games $\Gamma^{t}, t \in[0,1]$, with $\epsilon$ going to 0 if the mesh size $\delta$ of the triangulation $\Sigma$ goes to 0 . The result makes clear that it is possible to give an upper bound for $\delta$ in terms of $\epsilon$, the payoffs of the game, $R_{i}$, and the prior $p$.

\section{THE ALGORITHM}

Let a subset $B_{*}$ of $\Phi_{*}$ be given with the property that for every player $i$ there is at least one pure strategy $(i, k)$ in $B_{*}$. Such a set $B_{*}$ is called admissible. Let $B_{i}$ denote the set of all pure strategies of player $i$ in $B_{*}$. The set $B_{i}$ is related to all best replies of player $i$ against a certain strategy combination. The set $S\left(B_{*}\right)$ denotes all mixed strategy combinations that give positive weight only to pure strategies in $B_{*}$, so

$$
S\left(B_{*}\right)=\left\{s \in S \mid \sum_{(i, k) \in B_{i}} s_{i k}=1, i \in \mathcal{N}\right\} .
$$

Let a triangulation $\Sigma$ of $[0,1] \times S$ be given. For an admissible subset $B_{*}$ of $\Phi_{*}$, we denote by $\Sigma\left(B_{*}\right)$ the collection of $m$-faces of simplices in $\Sigma$, where $m=\left|B_{*}\right|+1-N$, that are contained in $[0,1] \times S\left(B_{*}\right)$, so

$$
\Sigma\left(B_{*}\right)=\left\{\tau \subset[0,1] \times S\left(B_{*}\right) \mid \exists \sigma \in \Sigma, \tau \text { is a }\left(\left|B_{*}\right|+1-N\right) \text {-face of } \sigma\right\} .
$$

Here $\left|B_{*}\right|$ denotes the cardinality of $B_{*}$. By repeated application of the result claiming that the relative boundary of a set is triangulated by the facets of a triangulation, it follows that $\Sigma\left(B_{*}\right)$ is a triangulation of $[0,1] \times$ $S\left(B_{*}\right)$. Notice that $\Sigma\left(\Phi_{*}\right)=\Sigma$.

Let an admissible $B_{*} \subset \Phi_{*}$ and a simplex $\sigma\left(x^{1}, \ldots, x^{m+1}\right) \in \Sigma\left(B_{*}\right)$ be given. Consider solutions $\xi=\left(\left(\lambda_{j}\right)_{j=1, \ldots, m+1},\left(\mu_{i k}\right)_{(i, k) \in \Phi_{*} \backslash B_{*}},\left(\beta_{i}\right)_{i \in \mathcal{N}}\right) \in$ $\mathbb{R}^{M+2}$ of the following system of equations:

$$
\begin{aligned}
& \sum_{j=1}^{m+1} \lambda_{j}=1, \\
& \sum_{j=1}^{m+1} \lambda_{j} \bar{G}_{i}\left(x^{j}\right)+\sum_{(i, k) \in \Phi_{i} \backslash B_{i}} \mu_{i k} e^{M_{i}}(k)-\beta_{i} 1^{M_{i}}=0^{M_{i}}, \quad i \in \mathcal{N} .
\end{aligned}
$$


If $\lambda_{j} \geq 0, j=1, \ldots, m+1$, and $\mu_{i k} \geq 0,(i, k) \in \Phi_{i} \backslash B_{i}$, then $\xi$ is called an admissible solution to Eq. (2). An admissible solution $\xi$ corresponds to an approximate Nash equilibrium $s$ of $\Gamma^{t}$. Indeed, $(t, s)=\sum_{j=1}^{m+1} \lambda_{j} x^{j}$, strategies in $B_{*}$ are best replies given the marginal payoff function $\bar{G}, \mu_{i k}$ is the payoff gap between strategy $(i, k) \in \Phi_{i} \backslash B_{i}$ and a best reply for player $i$, and $\beta_{i}$ is the payoff for player $i$ according to the marginal payoff function $\bar{G}$ when he uses a best reply. Since $\sigma \subset[0,1] \times S\left(B_{*}\right)$, strategies that are not a best reply are played with probability zero. An admissible solution to Eq. (2) is said to be degenerate if at least two of the variables $\lambda_{j}, j=1, \ldots, m+1$, and $\mu_{i k},(i, k) \in \Phi_{*} \backslash B_{*}$ are equal to 0 .

Later in this section, we introduce an algorithm that generates by means of lexicographic pivoting techniques a piecewise linear path of approximate Nash equilibria in $[0,1] \times S$ joining $\{0\} \times S$ to $\{1\} \times S$. The path is such that every $(t, s)$ on it corresponds to an admissible $B_{*} \subset \Phi_{*}$, a simplex $\sigma \in \Sigma\left(B_{*}\right)$, and an admissible solution $\xi$ as described earlier. The algorithm specifies in a unique way how to move from one simplex to another. For given $\left(B_{*}, \sigma\right)$, Eq. (2) corresponds to a linear system with $M+1$ equations and $M+2$ variables. If we rule out degeneracies, then a nonempty solution set is a one-dimensional compact line segment. The endpoints of the line segment are either approximate Nash equilibria for $\Gamma^{0}$ or $\Gamma^{1}$, or yield solutions for a new $\left(\bar{B}_{*}, \bar{\sigma}\right)$. Indeed, with degeneracies ruled out, at an endpoint either $\lambda_{j}=0$ for exactly one $j$ or $\mu_{i k}=0$ for exactly one $(i, k) \in \Phi_{i} \backslash B_{i}$. In the first case, the endpoint belongs to the facet $\tau$ of $\sigma$ opposite to the vertex $x^{j}$. If $\tau$ belongs to the relative interior of $[0,1] \times S\left(B_{*}\right)$, then there is a unique simplex $\bar{\sigma} \in \Sigma\left(B_{*}\right)$ such that $\bar{\sigma} \neq \sigma$, and $\tau$ is a facet of $\bar{\sigma}$. The algorithm continues by generating a line segment of solutions in $\bar{\sigma}$. If $\tau$ belongs to the relative boundary of $[0,1] \times S\left(B_{*}\right)$, then the endpoint is either an approximate Nash equilibrium for $\Gamma^{0}$, an approximate Nash equilibrium for $\Gamma^{1}$, or $\tau \in \Sigma\left(\bar{B}_{*}\right)$, with $\bar{B}_{*}$ a uniquely determined subset of $B_{*}$ with one less element, and the algorithm continues with a line segment of solutions in $\tau$. If $\mu_{i k}=0$, then strategy $(i, k)$ is also a best reply. The algorithm continues with a line segment of solutions in $\bar{\sigma}$, where $\bar{\sigma}$ is the unique simplex in $\Sigma\left(B_{*} \cup\{(i, k)\}\right)$ with $\sigma$ as a facet.

In game theory, degeneracy is not always a nongeneric phenomenon. For instance, for a normal-form representation of a game in extensive form, degeneracy is the rule rather than the exception, even if the payoffs in the extensive-form game are randomly chosen. But degeneracy also can easily occur in other normal-form games, representing certain economic situations, simply because payoffs are not randomly chosen but reflect some structure that is present in the economic model. We deal with degeneracy by exploiting lexicographic pivoting techniques. In this paper we extend the techniques used in Eaves (1971), Todd (1976a), Wright (1981), and Herings et al. (1996). 
Let us take a closer look at the system Eq. (2). For an admissible $B_{*} \subset \Phi_{*}$ and a facet $\tau\left(x^{1}, \ldots, x^{m}\right)$ of a simplex in $\Sigma\left(B_{*}\right)$, the $(M+1) \times(M+1)$ matrix $A_{B_{*}, \tau}$ is defined by

$$
A_{B_{*}, \tau}=\left[\begin{array}{ccccccccc}
1 & \cdots & 1 & 0 & \cdots & \cdots & \cdots & \cdots & 0 \\
& & & E_{1} & & 0 & -1^{M_{1}} & & 0 \\
\bar{G}\left(x^{1}\right) & \cdots & \bar{G}\left(x^{m}\right) & 0 & \ddots & 0 & 0 & \ddots & 0 \\
& & & 0 & 0 & E_{N} & 0 & 0 & -1^{M_{N}}
\end{array}\right],
$$

where, for $i \in \mathcal{N}, E_{i}=\left[e^{M_{i}}(k)\right]_{(i, k) \in \Phi_{i} \backslash B_{i}}$. The matrix $A_{B_{*}, \tau}$ corresponds to the coefficients in Eq. (2) when a facet $\tau$ of a simplex $\sigma$ is considered. Suppose that $A_{B_{*}, \tau}^{-1}$ exists. From $A_{B_{*}, \tau} A_{B_{*}, \tau}^{-1}=I^{M+1}$, it follows that the first column of $A_{B_{*}, \tau}^{-1}$ corresponds to an admissible solution to Eq. (2) for any $\sigma \in \Sigma\left(B_{*}\right)$ being the convex hull of $\tau$ and some vertex $x^{m+1} \in[0,1] \times S\left(B_{*}\right)$, whenever the first $M+1-N$ components of this column are nonnegative. No restrictions are imposed on the last $N$ rows of $A_{B_{*}, \tau}^{-1}$. In a nondegenerate solution, the first $M+1-N$ components are all positive, since $\lambda_{m+1}=0$ extends the solution for the facet $\tau$ to the simplex $\sigma$.

A row vector $x \in \mathbb{R}^{M+1}$ is lexicographically positive if $x \neq 0^{M+1^{\top}}$ and its first nonzero entry is positive. The matrix $A_{B_{*}, \tau}^{-1}$ is said to be semilexicopositive if each of the first $M+1-N$ rows is lexicographically positive.

Definition 4.1. Let $(\Gamma, p, \Sigma)$ be given and let $B_{*}$ be an admissible subset of $\Phi_{*}$. A facet $\tau$ of a simplex in $\Sigma\left(B_{*}\right)$ is $B_{*}$ complete if $A_{B_{*}, \tau}^{-1}$ exists and is semi-lexicopositive.

By restricting attention to complete facets, we can take care of degeneracy problems. Given a linear system of equations as in Eq. (2), determined by a $B_{*}$-complete facet $\tau$, we pivot in a uniquely determined new column of either the type $\left(1, \bar{G}\left(x^{m+1}\right)\right)$ or the type $\left(0, e^{M}(k)\right)$. Generically, such a pivot step determines in a unique way a column out of the first $M+1-N$ to be replaced. In degenerate cases, the leaving column may not be uniquely determined. A semi-lexicographic pivot step is a pivot step where the leaving column is selected in such a way that the inverse of the resulting matrix $A_{B_{*}, \tau}$ is semi-lexicopositive. We show that a semilexicographic pivot step determines in a unique way in all cases which column out of the first $M+1-N$ is to be replaced.

To make the notion of a semi-lexicographic pivot step more precise, we need the concept of a lexicographic ordering. A vector $\bar{x} \in \mathbb{R}^{n}$ is said to be lexicographically greater than a vector $x \in \mathbb{R}^{n}$ if $\bar{x}-x$ is lexicographically positive. In this way, the lexicographic ordering is obtained as a complete ordering on $\mathbb{R}^{n}$. Consider a $B_{*}$-complete facet $\tau$ and a column 
$z \in \mathbb{R}^{M+1}$ that has to be pivoted in the system determined by the matrix $A_{B_{*}, \tau}$. In all cases that we consider, $z$ is of either the type $\left(1, \bar{G}\left(x^{m+1}\right)\right)$ or the type $\left(0, e^{M}(k)\right)$. Define $y \in \mathbb{R}^{M+1}$ as $y=A_{B_{*}, \tau}^{-1} z$. We show in Lemmas 4.3 and 4.4 that $y_{j}>0$ for some $j \in\{1, \ldots, M+1-N\}$. Let $j^{\prime} \in\{1, \ldots, M+1-N\}$ be such that $\frac{1}{y_{j^{\prime}}}\left(A_{B_{*}, \tau}^{-1}\right)_{j^{\prime}}$. is minimal according to the lexicographic ordering over all row vectors $\frac{1}{y_{j}}\left(A_{B_{*}, \tau}^{-1}\right)_{j}$. for which $y_{j}>0$ and $j \in\{1, \ldots, M+1-N\}$. We show in Lemmas 4.3 and 4.4 that $j^{\prime}$ is uniquely determined. The operation of replacing column $j^{\prime}$ of $A_{B_{*}, \tau}$ by $z$ is a semi-lexicographic pivot step. A semi-lexicographic pivot step is not more difficult to carry out than a normal pivot step and coincides with it in nondegenerate cases.

The following lemma is well known in linear programming theory (see, e.g., Murty, 1983). It can be used to link several complete facets to each other.

Lemma 4.2. Let an invertible $n \times n$ matrix $A$, a vector $z$ of $\mathbb{R}^{n}$, and some $j \in\{1, \ldots, n\}$ be given. The $n \times n$ matrix $\bar{A}$ is defined by $\bar{A}=\left(A_{\cdot 1} \ldots A_{\cdot j-1} \quad z \quad A_{\cdot j+1} \ldots A_{\cdot n}\right)$. Then either $\left(A^{-1} z\right)_{j}=0$ and $\bar{A}$ is singular, or $\left(A^{-1} z\right)_{j} \neq 0$ and

$$
\bar{A}^{-1}=\left(\begin{array}{c}
\left(A^{-1}\right)_{1 \cdot}-\frac{\left(A^{-1} z\right)_{1}}{\left(A^{-1} z\right)_{j}}\left(A^{-1}\right)_{j} . \\
\vdots \\
\left(A^{-1}\right)_{j-1 \cdot}-\frac{\left(A^{-1} z\right)_{j-1}}{\left(A^{-1} z\right)_{j}}\left(A^{-1}\right)_{j} . \\
\frac{1}{\left(A^{-1} z\right)_{j}}\left(A^{-1}\right)_{j} . \\
\left(A^{-1}\right)_{j+1 \cdot}-\frac{\left(A^{-1} z\right)_{j+1}}{\left(A^{-1} z\right)_{j}}\left(A^{-1}\right)_{j} . \\
\vdots \\
\left(A^{-1}\right)_{n \cdot}-\frac{\left(A^{-1} z\right)_{n}}{\left(A^{-1} z\right)_{j}}\left(A^{-1}\right)_{j} .
\end{array}\right) .
$$

Lemma 4.2 is easily shown by calculating $\bar{A}^{-1} \bar{A}$.

Lemma 4.3 describes all possible cases that may occur if a $B_{*}$-complete facet $\tau$ is given and a semi-lexicographic pivot step with a vector $\left(1, \bar{G}\left(x^{m+1}\right)\right)$ is made, where $x^{m+1}$ is a vertex of a simplex having $\tau$ as a facet opposite to it. The structure of the proof of Lemma 4.3 (as well as Lemma 4.4) is closely related to the one of Wright (1981) and Herings et al. (1996), although, of course, we have to deal with the specifics of our algorithm. 
Lemma 4.3. Let $(\Gamma, p, \Sigma)$ be given. Consider a $B_{*}$-complete facet $\tau$ of a simplex $\sigma \in \Sigma\left(B_{*}\right)$. Then exactly one of the following cases holds:

1. $\sigma$ has exactly one other $B_{*}$-complete facet $\bar{\tau}$.

2. $\sigma$ is $\bar{B}_{*}$ complete for precisely one admissible $\bar{B}_{*} \subset \Phi_{*}$.

Proof. Let $x^{m+1}$ be the vertex of $\sigma$ not contained in $\tau$ and let $y \in \mathbb{R}^{M+1}$ be given by

$$
y=A_{B_{*}, \tau}^{-1}\left(\begin{array}{c}
1 \\
\bar{G}\left(x^{m+1}\right)
\end{array}\right) .
$$

Since $\left(A_{B_{*}, \tau}\right)_{1}=\left(1^{m^{\top}}, 0^{M+1-m^{\top}}\right)$, it holds that $\sum_{j=1}^{m}\left(A_{B_{*}, \tau}^{-1}\right)_{j 1}=1$ and $\sum_{j=1}^{m}\left(A_{B_{*}, \tau}^{-1}\right)_{j l}=0, l=2, \ldots, M+1$. Suppose that the first $M+1-N$ components of $y$ are nonpositive. Then we obtain a contradiction, since

$$
\begin{aligned}
0 \geq\left(1^{m^{\top}}, 0^{M+1-m^{\top}}\right) y & =\left(1^{m^{\top}}, 0^{M+1-m^{\top}}\right) A_{B_{*}, \tau}^{-1}\left(\begin{array}{c}
1 \\
\bar{G}\left(x^{m+1}\right)
\end{array}\right) \\
& =\left(1,0^{M^{\top}}\right)\left(\begin{array}{c}
1 \\
\bar{G}\left(x^{m+1}\right)
\end{array}\right)=1,
\end{aligned}
$$

where the first inequality uses that $m \leq M+1-N$. Consequently, $y_{j}>0$ for some $j \in\{1, \ldots, M+1-N\}$. Let $j^{\prime} \in\{1, \ldots, M+1-N\}$ be the column in $A_{B_{*}}, \tau$ pivoted out by a semi-lexicographic pivot step, so $j^{\prime}$ is such that $\frac{1}{y_{j^{\prime}}}\left(A_{B_{*}, \tau}^{-1}\right)_{j^{\prime}}$. is minimal according to the lexicographic ordering over all row vectors $\frac{1}{y_{j}}\left(A_{B_{*}, \tau}^{-1}\right)_{j}$. for which $y_{j}>0$ and $j \in\{1, \ldots, M+1-N\}$. The row $j^{\prime}$ is uniquely determined, because otherwise there would be two rows that are linearly dependent, and $A_{B_{*}, \tau}^{-1}$ would not be invertible. It holds that either $j^{\prime} \in\{1, \ldots, m\}$ or $j^{\prime} \in\{m+1, \ldots, M+1-N\}$.

In the first case, let $\bar{\tau}$ be the facet of $\sigma$ opposite $x^{j^{\prime}}$. By Lemma 4.2 and the choice of $j^{\prime}$, it follows that $A_{B_{*}, \bar{\tau}}^{-1}$ exists and is semi-lexicopositive. So $\bar{\tau}$ is $B_{*}$ complete.

In the second case, it holds that column $j^{\prime}$ of $A_{B_{*}, \tau}$ corresponds to pure strategy $k$ of player $i$. Let $\bar{B}_{*} \subset \Phi_{*}$ be defined by $\bar{B}_{*}^{*}=B_{*} \cup\{(i, k)\}$. Obviously, $\bar{B}_{*}$ is admissible, and $\sigma$ is a facet of a simplex of $\Sigma\left(\bar{B}_{*}\right)$. By Lemma 4.2 and the choice of $j^{\prime}$, it follows that $A_{\bar{B}_{*}, \sigma}^{-1}$ exists and is semi-lexicopositive. So $\sigma$ is $\bar{B}_{*}$ complete.

Lemma 4.2 guarantees that replacement of another column of $A_{B_{*}}, \tau$ would give a new matrix that does not have a semi-lexicopositive inverse. This implies that the two cases considered here are mutually exclusive, and that the facet $\bar{\tau}$ and the set $\bar{B}_{*}$ are uniquely determined.

Consider a $B_{*}$-complete facet $\tau$ that is also a simplex belonging to $\Sigma\left(\bar{B}_{*}\right)$ for some admissible $\bar{B}_{*} \subset \Phi_{*}$. Then there is a unique strategy $(\bar{i}, \bar{k})$ such 
that $B_{*}=\bar{B}_{*} \cup\{(\bar{i}, \bar{k})\}$. Lemma 4.4 describes all possible cases that may occur if a semi-lexicographic pivot step with a vector $\left(0, e^{M}(l)\right)$ is made, where $l=\sum_{i=1}^{\bar{i}-1} M_{i}+\bar{k}$.

Lemma 4.4. Let $(\Gamma, p, \Sigma)$ be given. Consider a $B_{*}$-complete facet $\tau$ that belongs to $\Sigma\left(\bar{B}_{*}\right)$ for some admissible $\bar{B}_{*} \subset \Phi_{*}$. Then exactly one of the following cases holds:

1. $\tau$ is $\widehat{B}_{*}$ complete for precisely one admissible $\widehat{B}_{*} \subset \Phi_{*}$ with $\widehat{B}_{*} \neq B_{*}$.

2. Precisely one facet $v$ of $\tau$ is $\bar{B}_{*}$ complete.

Proof. There is a unique strategy $(\bar{i}, \bar{k})$ such that $B_{*}=\bar{B}_{*} \cup\{(\bar{i}, \bar{k})\}$. Define $l=\sum_{i=1}^{\bar{i}-1} M_{i}+\bar{k}$. Let $y \in \mathbb{R}^{M+1}$ be given by

$$
y=A_{B_{*}, \tau}^{-1}\left(\begin{array}{c}
0 \\
e^{M}(l)
\end{array}\right) .
$$

Since $\left(A_{B_{*}, \tau}\right)_{1}=\left(1^{m^{\top}}, 0^{M+1-m^{\top}}\right)$, it holds that $\sum_{j=1}^{m}\left(A_{B_{*}, \tau}^{-1}\right)_{j 1}=1$ and $\sum_{j=1}^{m}\left(A_{B_{*}, \tau}^{-1}\right)_{j l}=0, l=2, \ldots, M+1$. Suppose that the first $M+1-N$ components of $y$ are nonpositive. Then

$$
\begin{aligned}
\left(1^{m^{\top}}, 0^{M+1-m^{\top}}\right) y & =\left(1^{m^{\top}}, 0^{M+1-m^{\top}}\right) A_{B_{*}, \tau}^{-1}\left(\begin{array}{c}
0 \\
e^{M}(l)
\end{array}\right) \\
& =\left(1,0^{M^{\top}}\right)\left(\begin{array}{c}
0 \\
e^{M}(l)
\end{array}\right)=0,
\end{aligned}
$$

so the first $m$ components of $y$ are 0 . Now it holds that

$$
1=\sum_{j=1}^{M+1}\left(A_{B_{*}, \tau}\right)_{l+1, j} y_{j}=\sum_{j=m+1}^{M+1}\left(A_{B_{*}, \tau}\right)_{l+1, j} y_{j}=-y_{M+1-N+\bar{i}} .
$$

So $y_{M+1-N+\bar{i}}=-1$, and a solution to $A_{B_{*}, \tau} y=\left(\begin{array}{c}0 \\ e^{M}(l)\end{array}\right)$ can exist only if $(\bar{i}, k) \notin B_{\bar{i}}$ for every $k \neq \bar{k}$. Since $B_{*}=\bar{B}_{*} \cup\{(\bar{i}, \bar{k})\}$, it follows that $\bar{B}_{*}$ is not admissible, a contradiction. Consequently, $y_{j}>0$ for some $j \in\{1, \ldots, M+$ $1-N\}$. Let $j^{\prime} \in\{1, \ldots, M+1-N\}$ be the column in $A_{B_{*}, \tau}$ pivoted out by a semi-lexicographic pivot step, so $j^{\prime}$ is such that $\frac{1}{y_{j^{\prime}}}\left(A_{B_{*}, \tau}^{-1}\right)_{j^{\prime}}$. is minimal according to the lexicographic ordering over all row vectors $\frac{1}{y_{j}}\left(A_{B_{*}, \tau}^{-1}\right)_{j}$. for which $y_{j}>0$ and $j \in\{1, \ldots, M+1-N\}$. The row $j^{\prime}$ must be unique, because otherwise $A_{B_{*}, \tau}^{-1}$ would not be invertible. It holds that either $j^{\prime} \in$ $\{1, \ldots, m\}$ or $j^{\prime} \in\{m+1, \ldots, M+1-N\}$.

If $j^{\prime} \in\{m+1, \ldots, M+1-N\}$, then let $\left(i^{\prime}, k^{\prime}\right)$ be the strategy that corresponds to column $j^{\prime}$. Let an admissible $\widehat{B}_{*} \subset \Phi_{*}$ be defined by 
$\widehat{B}_{*}=\bar{B}_{*} \cup\left\{\left(i^{\prime}, k^{\prime}\right)\right\}$, and consider $A_{\widehat{B}_{*}, \tau}$. By Lemma 4.2 , the choice of $j^{\prime}$ guarantees that $A_{\widehat{B}_{*}, \tau}^{-1}$ is semi-lexicopositive, and thus $\tau$ is $\widehat{B}_{*}$ complete.

If $j^{\prime} \in\{1, \ldots, m\}$, then let $v$ be the facet of $\tau$ opposite $x^{j^{\prime}}$. Using Lemma 4.2 , the choice of $j^{\prime}$ implies that $A_{\bar{B}_{*}, v}^{-1}$ is semi-lexicopositive and hence $v$ is $\bar{B}_{*}$ complete.

Again, Lemma 4.2 guarantees that replacing another column of $A_{B_{*}, \tau}$ would give a new matrix that does not have a semi-lexicopositive inverse. This implies that the two cases considered here are mutually exclusive, and that the facet $v$ and the set $\widehat{B}_{*}$ are uniquely determined.

The consideration of $B_{*}$-complete facets will also provide us with a unique, endogenously determined starting point for the algorithm. The admissible subset $B_{*}^{0}$ of $\Phi_{*}$ is defined by $B_{i}^{0}=\left\{\left(i, k_{i}\right)\right\}, i \in \mathcal{N}$, where $k_{i}$ is the largest integer such that $\left(i, k_{i}\right)$ is a best reply to $p$ for player $i$. Notice that $\left|B_{*}^{0}\right|=N$. The facet (vertex) $\tau=\{0\} \times S\left(B_{*}^{0}\right)$ is $B_{*}^{0}$ complete, (see Lemma 6.1 for a proof). It will be shown that there is no other $B_{*}$-complete facet $\tau$ in $\{0\} \times S$. Even in degenerate cases, the semi-lexicographic rules single out the unique $B_{*}^{0}$-complete facet $\{0\} \times S\left(B_{*}^{0}\right)$, which will serve as a unique, endogenously determined starting point of the algorithm.

The steps of the algorithm are as follows.

Algorithm 4.5. Let $(\Gamma, p, \Sigma)$ be given.

Step 0 . Let $m=1$ and $n=1$. Let $B_{*}=B_{*}^{0}, \tau^{1}=\{0\} \times S\left(B_{*}\right)$, and $x^{2}$ be the unique vertex of the 1 -simplex of $\Sigma\left(B_{*}\right)$ containing $\tau^{1}$ as the facet opposite to it.

Step 1. Let $\sigma$ be equal to the convex hull of $\tau^{n}$ and $\left\{x^{m+1}\right\}$. Make a semi-lexicographic pivot step with $\left(1, \bar{G}\left(x^{m+1}\right)\right)$ into the system of equations Eq. (2) corresponding to $A_{B_{*}, \tau^{n}}$, yielding a unique column $j^{\prime}$ of $A_{B_{*}, \tau^{n}}$ which has to be replaced. If $j^{\prime} \in\{m+1, \ldots, M+1-N\}$, then go to step 3 with $\left(i^{\prime}, k^{\prime}\right)$ the pure strategy corresponding to column $j^{\prime}$. Otherwise, go to step 2.

Step 2. Increase the value of $n$ by 1 , and let $\tau^{n}$ be the facet of $\sigma$ opposite $x^{j^{\prime}}$. If $\tau^{n} \subset\{1\} \times S$, then the algorithm terminates with an approximate Nash equilibrium $s^{*}$ of $\Gamma^{1}$ induced by the solution of Eq. (2) corresponding to $A_{B_{*}, \tau^{n}}$. If $\tau^{n} \in \Sigma\left(\bar{B}_{*}\right)$ for some admissible $\bar{B}_{*} \subset \Phi_{*}$, then go to step 4 . Otherwise, there is exactly one $m$-simplex $\bar{\sigma}$ of $\Sigma\left(B_{*}\right)$ such that $\bar{\sigma} \neq \sigma$ and $\tau^{n}$ is a facet of $\bar{\sigma}$. Go to step 1 with $x^{m+1}$ as the unique vertex of $\bar{\sigma}$ opposite $\tau^{n}$.

Step 3. Let the admissible $\bar{B}_{*} \subset \Phi_{*}$ be defined by $\bar{B}_{*}=B_{*} \cup\left\{\left(i^{\prime}, k^{\prime}\right)\right\}$. There is a unique simplex $\bar{\sigma}$ of $\Sigma\left(\bar{B}_{*}\right)$ having $\sigma$ as a facet. Increase the value of both $m$ and $n$ by 1 and go to step 1 with $x^{m+1}$ as the unique vertex of $\bar{\sigma}$ opposite $\sigma, B_{*}=\bar{B}_{*}$, and $\tau^{n}=\sigma$. 
Step 4. Let $\sigma$ be equal to $\tau^{n}$. Make a semi-lexicographic pivot step with $\left(0, e^{M}\left(\sum_{i=1}^{\bar{i}-1} M_{i}+\bar{k}\right)\right)$ into the system of equations in Eq. (2) corresponding to $A_{B_{*}, \tau^{n}}$, where $(\bar{i}, \bar{k})$ is such that $\bar{B}_{*} \cup\{(\bar{i}, \bar{k})\}=B_{*}$. This yields a unique column $j^{\prime}$ of $A_{B_{*}, \tau^{n}}$ which has to be replaced. If $j^{\prime} \in$ $\{m+1, \ldots, M+1-N\}$, then decrease the value of both $m$ and $n$ by 1 and go to step 3 with $\left(i^{\prime}, k^{\prime}\right)$ the pure strategy corresponding to column $j^{\prime}$ and $B_{*}=\bar{B}_{*}$. Otherwise, decrease the value of $m$ by 1 and go to step 2 with $B_{*}=\bar{B}_{*}$.

The operation used in the proof of Lemma 4.3, where a column of $A_{B_{*}, \tau}$ is determined in a unique way and is replaced by the vector $\left(1, \bar{G}\left(x^{m+1}\right)\right)$, corresponds to the semi-lexicographic pivot step made in step 1 of Algorithm 4.5. Case 1 of Lemma 4.3 occurs if one goes from step 1 of Algorithm 4.5 to step 2, and case 2 occurs if one goes from step 1 to step 3.

The operation used in the proof of Lemma 4.4, where a column of $A_{B_{*}, \tau}$ is determined in a unique way and is replaced by the vector $\left(0, e^{M}\left(\sum_{i=1}^{\bar{i}-1} M_{i}+\bar{k}\right)^{\top}\right)^{\top}$, corresponds to the semi-lexicographic pivot step performed in step 4 of Algorithm 4.5. Case 1 of Lemma 4.4 happens if one goes from step 4 of Algorithm 4.5 to step 3, and case 2 happens if one goes from step 4 to step 2 .

In Section 6 it is shown that the algorithm terminates after a finite number of steps, after having generated a $B_{*}$-complete facet $\tau$ that is a subset of $\{1\} \times S\left(B_{*}\right)$.

Consider all different pairs $\left(B_{*}^{1}, \tau^{1}\right), \ldots,\left(B_{*}^{\hat{k}}, \tau^{\hat{k}}\right)$ successively generated by Algorithm 4.5. For $k=1, \ldots, \hat{k}, \tau^{k}=\tau^{k}\left(x^{1}, \ldots, x^{m^{k}}\right)$, with $m^{k}=\left|B_{*}^{k}\right|+$ $1-N$. Define $\tilde{x}^{k}=\left(\tilde{t}^{k}, \tilde{s}^{k}\right) \in \tau^{k}$ by $\tilde{x}^{k}=\sum_{j=1}^{m^{k}} \bar{\lambda}_{j} x^{j}$, with $\bar{\lambda}_{j}$ following from the admissible solution of the system in Eq. (2) corresponding to $A_{B_{*}^{k}, \tau^{k}}$. So $\tilde{s}^{k}$ is an approximate Nash equilibrium of $\Gamma^{\tilde{\tau}^{k}}$. For $r \in \mathbb{R}$, define $\lfloor r\rfloor$ as the greatest integer which is less than or equal to $r$. Finally, define the piecewise linear continuous function $\pi:[0,1] \rightarrow[0,1] \times S$ by

$$
\begin{aligned}
\pi(r)= & (1-(\hat{k}-1) r+\lfloor(\hat{k}-1) r\rfloor) \tilde{x}^{1+\lfloor(\hat{k}-1) r\rfloor} \\
& +((\hat{k}-1) r-\lfloor(\hat{k}-1) r\rfloor) \tilde{x}^{2+\lfloor(\hat{k}-1) r\rfloor .}
\end{aligned}
$$

For $r=1$, the definition of $\pi(r)$ reads as $\pi(1)=1 \cdot \tilde{x}^{\hat{k}}+0 \cdot \tilde{x}^{\hat{k}+1}$. We do not formally define the point $\tilde{x}^{\hat{k}+1}$, because the exact definition is immaterial.

Consider some $r \in[0,1]$. Then $\pi(r)=(t, s)$ is a convex combination of two points $\tilde{x}^{k}$ and $\tilde{x}^{k+1}$. It is easily verified, because of the linearity of the system, that $\pi(r)$ gives rise to an admissible solution for Eq. (2) with $B_{*}$ equal to $B_{*}^{k} \cap B_{*}^{k+1}$ and $\sigma$ equal to the convex hull of $\tau^{k}$ and $\tau^{k+1}$. So $s$ is an approximate Nash equilibrium of $\Gamma^{t}$. The function $\pi$ : $[0,1] \rightarrow[0,1] \times S$ is called the path generated by the algorithm. When 
the mesh size of the triangulation used goes to 0 , the path generated by the algorithm converges to the linear tracing procedure. For a proof and a precise statement, see Section 7.

\section{AN EXAMPLE}

To illustrate Algorithm 4.5, we consider the game of Figure 2. The unique Nash equilibrium of this game is $\left(\left(\frac{2}{3}, \frac{1}{3}\right),\left(\frac{3}{4}, \frac{1}{4}\right)\right)$. We write $\Phi_{1}=\{(1,1),(1,2)\}, \Phi_{2}=\{(2,1),(2,2)\}, R_{1}((1,1),(2,1))=2$, etc. Consider a prior $p=\left(\left(\frac{1}{2}, \frac{1}{2}\right),\left(\frac{3}{4}, \frac{1}{4}\right)\right)$. It follows that

$$
\begin{aligned}
& G_{11}(t, s)=t\left(2 s_{21}+s_{22}\right)+(1-t) \frac{7}{4}, \\
& G_{12}(t, s)=t\left(s_{21}+4 s_{22}\right)+(1-t) \frac{7}{4}, \\
& G_{21}(t, s)=t\left(2 s_{11}+4 s_{12}\right)+(1-t) 3,
\end{aligned}
$$

and

$$
G_{22}(t, s)=t\left(4 s_{11}\right)+(1-t) 2 .
$$

We take the triangulation $\Sigma$ depicted in Figure 1.

First, we have to determine $B_{*}^{0}$. It is given by $B_{*}^{0}=\{(1,2),(2,1)\}$. Against the prior, both pure strategies give player 1 a payoff of $\frac{7}{4}$, $G_{11}\left(0,\left(\left(\frac{1}{2}, \frac{1}{2}\right),\left(\frac{3}{4}, \frac{1}{4}\right)\right)\right)=G_{12}\left(0,\left(\left(\frac{1}{2}, \frac{1}{2}\right),\left(\frac{3}{4}, \frac{1}{4}\right)\right)\right)=\frac{7}{4}$. The tie-breaking rule introduced to determine $B_{*}^{0}$ requires that the second pure strategy of player 1 be selected. It is obvious that $s^{0}=((0,1),(1,0))$ is a Nash equilibrium of $\Gamma^{0}$. The simplex $\tau^{1}$ is equal to $\{0\} \times\{((0,1),(1,0))\}$ and is denoted in Figure 1 by $\bar{x}^{3}$. The unique simplex of $\Sigma(\{(1,2),(2,1)\})$ containing $\tau^{1}$ as a facet is given by $\sigma=\operatorname{co}\left(\left\{\bar{x}^{3}, \bar{x}^{12}\right\}\right)$, and the unique vertex

\begin{tabular}{c|c|c|} 
& $\mathrm{L}$ & $\mathrm{R}$ \\
\hline $\mathrm{T}$ & 2,2 & 1,4 \\
\hline $\mathrm{B}$ & 1,4 & 4,0 \\
\hline
\end{tabular}

FIG. 2. A two-person game. 
of it not in $\tau^{1}$ is $\bar{x}^{12}$. This terminates step 0 . It holds that

$$
\begin{aligned}
A_{B_{*}^{0}, \tau^{1}} & =\left[\begin{array}{rrrrr}
1 & 0 & 0 & 0 & 0 \\
\frac{7}{4} & 1 & 0 & -1 & 0 \\
\frac{7}{4} & 0 & 0 & -1 & 0 \\
3 & 0 & 0 & 0 & -1 \\
2 & 0 & 1 & 0 & -1
\end{array}\right] \quad \text { and } \\
\left(A_{B_{*}^{0}, \tau^{1}}\right)^{-1} & =\left[\begin{array}{rrrrr}
1 & 0 & 0 & 0 & 0 \\
0 & 1 & -1 & 0 & 0 \\
1 & 0 & 0 & -1 & 1 \\
\frac{7}{4} & 0 & -1 & 0 & 0 \\
3 & 0 & 0 & -1 & 0
\end{array}\right] .
\end{aligned}
$$

It is easily verified that $\left(A_{B_{*}^{0}, \tau^{1}}\right)^{-1}$ is semi-lexicopositive. The first column of $\left(A_{B_{*}^{0}, \tau^{1}}\right)^{-1}$, given by the vector $\left(1,0,1, \frac{7}{4}, 3\right)$, corresponds to an admissible solution of Eq. (2). Here $\lambda_{1}=1$, the weight attached to vertex $\bar{x}^{3}$; $\mu_{11}=0$ and $\mu_{22}=1$, the losses in payoff for player 1 using his first strategy and for player 2 using his second strategy; and $\beta_{1}=\frac{7}{4}$ and $\beta_{2}=3$, the payoffs of players 1 and 2 . Now we have to make a semi-lexicographic pivot step with $\left(1, \bar{G}\left(\bar{x}^{12}\right)\right)=\left(1,\left(\frac{15}{8}, \frac{11}{8}, \frac{7}{2}, 1\right)\right)$ into the system of equations in Eq. (2) corresponding to $A_{B_{*}^{0}, \tau^{1}}$. This yields column 2 to be replaced, which corresponds to pure strategy $(1,1)$. Since $2 \in\{2,3\}$, we go to step 3 and increase the dimension of the simplices generated by 1 .

Step 3 takes $\bar{B}_{*}=B_{*} \cup\{(1,1)\}=\{(1,1),(1,2),(2,1)\}$. The unique simplex $\bar{\sigma}$ of $\Sigma\left(\bar{B}_{*}\right)$ having $\operatorname{co}\left(\left\{\bar{x}^{3}, \bar{x}^{12}\right\}\right)$ as a facet is $\operatorname{co}\left(\left\{\bar{x}^{2}, \bar{x}^{3}, \bar{x}^{12}\right\}\right)$. Next, $m$ and $n$ are set equal to 2 , and step 1 is carried out with $x^{3}=\bar{x}^{2}$, $B_{*}=\{(1,1),(1,2),(2,1)\}, \tau^{2}=\operatorname{co}\left(\left\{\bar{x}^{3}, \bar{x}^{12}\right\}\right)$, etc. The algorithm reaches $\{1\} \times S$ after generating the 13 facets that are denoted by $\tau^{1}, \ldots, \tau^{13}$ in Figure 3 .

It can be verified that $\tau^{1}=\left\{\bar{x}^{3}\right\}, \tau^{2}=\operatorname{co}\left(\left\{\bar{x}^{3}, \bar{x}^{12}\right\}\right), \tau^{3}=\operatorname{co}\left(\left\{\bar{x}^{2}, \bar{x}^{12}\right\}\right)$, $\tau^{4}=\operatorname{co}\left(\left\{\bar{x}^{2}, \bar{x}^{11}\right\}\right), \tau^{5}=\operatorname{co}\left(\left\{\bar{x}^{2}, \bar{x}^{10}\right\}\right), \tau^{6}=\operatorname{co}\left(\left\{\bar{x}^{1}, \bar{x}^{10}\right\}\right), \tau^{7}=\operatorname{co}\left(\left\{\bar{x}^{4}\right.\right.$, $\left.\left.\bar{x}^{10}\right\}\right), \tau^{8}=\operatorname{co}\left(\left\{\bar{x}^{4}, \bar{x}^{10}, \bar{x}^{13}\right\}\right), \tau^{9}=\operatorname{co}\left(\left\{\bar{x}^{10}, \bar{x}^{13}, \bar{x}^{14}\right\}\right), \tau^{10}=\operatorname{co}\left(\left\{\bar{x}^{10}, \bar{x}^{14}\right.\right.$, $\left.\left.\bar{x}^{23}\right\}\right), \tau^{11}=\operatorname{co}\left(\left\{\bar{x}^{10}, \bar{x}^{11}, \bar{x}^{23}\right\}\right), \tau^{12}=\operatorname{co}\left(\left\{\bar{x}^{11}, \bar{x}^{19}, \bar{x}^{23}\right\}\right)$, and $\tau^{13}=$ $\operatorname{co}\left(\left\{\bar{x}^{19}, \bar{x}^{20}, \bar{x}^{23}\right\}\right)$. An interesting situation occurs at $\tau^{6}$. In step 2 we reach the case where $\tau^{6}$ belongs to the Cartesian product of $[0,1]$ and the boundary of $S\left(B_{*}\right), \tau^{6} \in \Sigma(\{(1,1),(2,1)\})$. We have to go to step 4 to decrease the dimension by 1 . In step 4 we pivot in the vector $\left(0, e^{4}(2)\right)$, since $B_{*}=\bar{B}_{*} \cup\{(1,2)\}$. This yields column 3 to be replaced, which corresponds to pure strategy 2 of player 2 . Since $3 \in\{3\}$, we go to step 3 to 


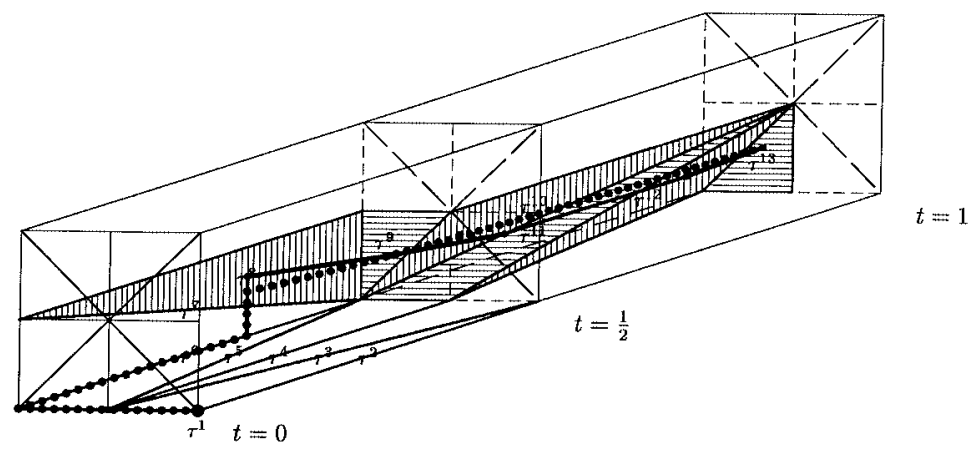

FIG. 3. The algorithm in action.

increase the dimension. It holds that $\tau^{6}$ is both $\{(1,1),(1,2),(2,1)\}$ complete and $\{(1,1),(2,1),(2,2)\}$ complete, yielding two different admissible solutions to the system of equations in Eq. (2).

The importance of the semi-lexicographic pivot steps becomes clear when reaching $\tau^{10}$. When $\bar{x}^{23}$ is pivoted in, ordinary pivot steps cannot determine whether $\bar{x}^{13}$ or $\bar{x}^{14}$ should be pivoted out. We denote the facet obtained by pivoting out $\bar{x}^{14}$ by $\bar{\tau}^{10}$, so $\bar{\tau}^{10}=\operatorname{co}\left(\left\{\bar{x}^{10}, \bar{x}^{13}, \bar{x}^{23}\right\}\right)$. In both cases, $B_{*}=\Phi_{*}$. It holds that

$$
\begin{aligned}
A_{\Phi_{*}, \tau^{10}} & =\left[\begin{array}{rrrrr}
1 & 1 & 1 & 0 & 0 \\
\frac{13}{8} & \frac{15}{8} & \frac{3}{2} & -1 & 0 \\
\frac{17}{8} & \frac{11}{8} & \frac{5}{2} & -1 & 0 \\
3 & \frac{5}{2} & 3 & 0 & -1 \\
2 & 3 & 2 & 0 & -1
\end{array}\right] \quad \text { and } \\
\left(A_{\Phi_{*}, \tau^{10}}\right)^{-1}= & {\left[\begin{array}{rrrrr}
0 & 2 & -2 & 2 & -2 \\
\frac{2}{3} & 0 & 0 & -\frac{2}{3} & \frac{2}{3} \\
\frac{1}{3} & -2 & 2 & -\frac{4}{3} & \frac{4}{3} \\
\frac{7}{4} & -\frac{3}{4} & -\frac{1}{4} & 0 & 0 \\
\frac{8}{3} & 0 & 0 & -\frac{2}{3} & -\frac{1}{3}
\end{array}\right] }
\end{aligned}
$$

and

$$
A_{\Phi_{*}, \bar{\tau}^{10}}=\left[\begin{array}{rrrrr}
1 & 1 & 1 & 0 & 0 \\
\frac{3}{2} & \frac{15}{8} & \frac{13}{8} & -1 & 0 \\
\frac{5}{2} & \frac{11}{8} & \frac{17}{8} & -1 & 0 \\
3 & \frac{5}{2} & \frac{5}{2} & 0 & -1 \\
2 & 3 & 3 & 0 & -1
\end{array}\right] \quad \text { and }
$$




$$
\left(A_{\Phi_{*}, \bar{\tau}^{10}}\right)^{-1}=\left[\begin{array}{rrrrr}
\frac{1}{3} & 0 & 0 & \frac{2}{3} & -\frac{2}{3} \\
\frac{2}{3} & 1 & -1 & \frac{1}{3} & -\frac{1}{3} \\
0 & -1 & 1 & -1 & 1 \\
\frac{7}{4} & -\frac{3}{4} & -\frac{1}{4} & 0 & 0 \\
\frac{8}{3} & 0 & 0 & -\frac{2}{3} & -\frac{1}{3}
\end{array}\right] .
$$

It is easily verified that $\left(A_{\Phi_{*}, \bar{\tau}^{10}}\right)^{-1}$ is not semi-lexicopositive, since the third row is not lexicographically positive. So $\bar{\tau}^{10}$ will not be generated by the semi-lexicographic pivot steps, but instead $\tau^{10}$ is the uniquely determined facet. Since $\tau^{13} \subset\{1\} \times S$, the algorithm will terminate there, as is dictated by step 2 .

The path generated by the algorithm in the example is illustrated in Figure 3 by the heavily drawn line going from $\tau^{1}$ to $\tau^{13}$. It is given by the points

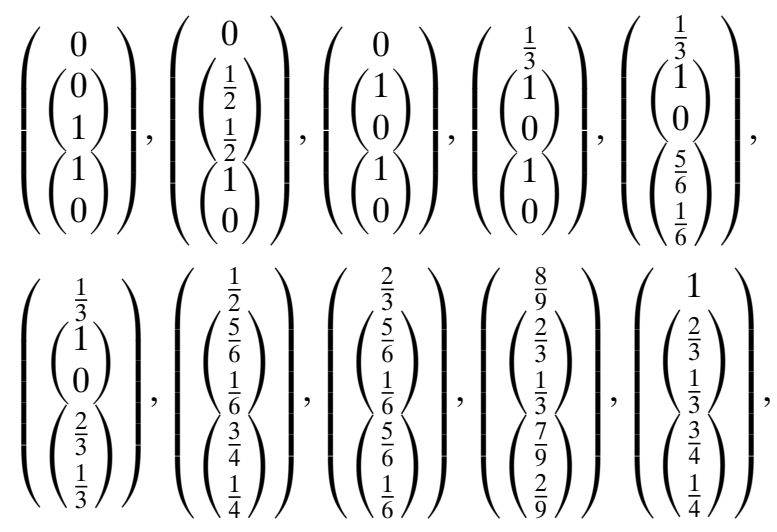

and all convex combinations of two successive points. The endpoint is exactly equal to the unique Nash equilibrium of the game $\Gamma$.

Because the game of Figure 2 is so simple, it is possible to determine the set $\mathscr{L}$ exactly. This set is depicted in Figure 3 by the dotted line. It is easily seen that $\mathscr{L}$ consists of only one component and $\mathscr{L}=\mathscr{L}^{\mathrm{c}}$. It is given by the points

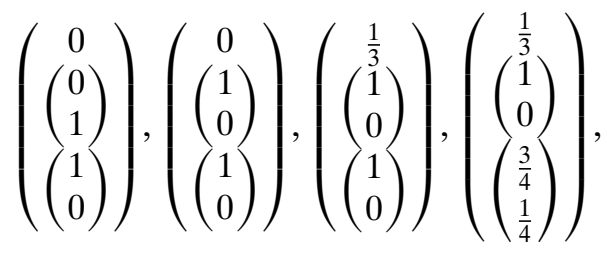


all convex combinations of two successive points, and also the points

$$
\left(\begin{array}{c}
t \\
\left(\begin{array}{c}
\frac{1+3 t}{6 t} \\
\frac{3 t-1}{6 t}
\end{array}\right) \\
\left(\begin{array}{c}
\frac{3}{4} \\
\frac{1}{4}
\end{array}\right)
\end{array}\right), \quad t \in\left(\frac{1}{3}, 1\right] .
$$

It is clear from the figure that even though the mesh size of the triangulation is fairly large, the set $\mathscr{L}$ is approximated very well by Algorithm 4.5. The point finally computed, $\tilde{x}^{13}=\left(1,\left(\left(\frac{2}{3}, \frac{1}{3}\right),\left(\frac{3}{4}, \frac{1}{4}\right)\right)\right)$, yields the exact Nash equilibrium $s=\left(\left(\frac{2}{3}, \frac{1}{3}\right),\left(\frac{3}{4}, \frac{1}{4}\right)\right)$.

\section{CONVERGENCE TO AN $\epsilon$-NASH EQUILIBRIUM OF $\Gamma$}

We show that every step in Algorithm 4.5 is unique and feasible, and that the algorithm generates a piecewise linear path in a sequence of adjacent simplices. The path consists of approximate Nash equilibria of $\Gamma^{t}, t \in[0,1]$. First, we show that $\bar{\tau}=\{0\} \times S\left(B_{*}^{0}\right)$ is $B_{*}^{0}$ complete and that there is no other 0 -simplex in the slice $\{0\} \times S$ that is $B_{*}$ complete for some $B_{*}$. We consider the case $\left|B_{*}\right|=N$ first.

Lemma 6.1. Let $(\Gamma, p, \Sigma)$ be given. The facet $\bar{\tau}=\{0\} \times S\left(B_{*}^{0}\right)$ is $B_{*}^{0}$ complete. There is no other $B_{*}$-complete facet $\tau$ in $\{0\} \times S$ with $\left|B_{*}\right|=N$.

Proof. Let $t=0$ and $\left|B_{*}\right|=N$. If $\tau \subset\{0\} \times S$ is $B_{*}$ complete, then, for $i \in \mathcal{N}, B_{i}=\left\{\left(i, k_{i}\right)\right\}$ with $\left(i, k_{i}\right)$ a best reply for player $i$ against $p$. It is straightforward that

$$
A_{B_{*}, \tau}=\left[\begin{array}{ccccccc}
1 & 0 & \ldots & 0 & 0 & \ldots & 0 \\
R_{1}(p \backslash(1,1)) & & & & & & \\
\vdots & E_{1} & 0 & 0 & -1^{M_{1}} & 0 & 0 \\
R_{1}\left(p \backslash\left(1, M_{1}\right)\right) & & & & & & \\
\vdots & 0 & \ddots & 0 & 0 & \ddots & 0 \\
R_{N}(p \backslash(N, 1)) & & & & & & \\
\vdots & 0 & 0 & E_{N} & 0 & 0 & -1^{M_{N}}
\end{array}\right],
$$


where $E_{i}$ is the $M_{i} \times M_{i}$ unit matrix with column $e^{M_{i}}\left(k_{i}\right)$ deleted. Inverting $A_{B_{*}, \tau}$, we find that

$$
A_{B_{*}, \tau}^{-1}=\left[\begin{array}{cccc}
1 & 0 & \cdots & 0 \\
\widetilde{R}_{1} & \widetilde{E}_{1} & 0 & 0 \\
\vdots & 0 & \ddots & 0 \\
\widetilde{R}_{N} & 0 & 0 & \widetilde{E}_{N} \\
R_{1}\left(p \backslash\left(1, k_{1}\right)\right) & & & \\
\vdots & -e^{N \times M_{1}}\left(1, k_{1}\right) & \cdots & -e^{N \times M_{N}}\left(N, k_{N}\right)
\end{array}\right],
$$

where $\widetilde{R}_{i} \in \mathbb{R}_{+}^{M_{i}-1}$ is the column vector given by $\widetilde{R}_{i l}=R_{i}\left(p \backslash\left(i, k_{i}\right)\right)$ $R_{i}(p \backslash(i, l)),(i, l) \in \Phi_{i} \backslash\left\{\left(i, k_{i}\right)\right\}$, and where $\widetilde{E}_{i}$ is the $\left(M_{i}-1\right) \times M_{i}$ matrix given by

$$
\widetilde{E}_{i}=\left[e^{M_{i}-1}(1) \cdots e^{M_{i}-1}\left(k_{i}-1\right)-1^{M_{i}-1} e^{M_{i}-1}\left(k_{i}\right) \cdots e^{M_{i}-1}\left(M_{i}-1\right)\right]
$$

and $e^{N \times M_{i}}\left(i, k_{i}\right)$ is the $\left(N \times M_{i}\right)$ matrix filled with 0 's except in row $i$ column $k_{i}$, where a 1 occurs. If $k_{i}=1$, then the first column of $\widetilde{E}_{i}$ is given by $-1^{M_{i}-1}$, and if $k_{i}=M_{i}$, then the last column of $\widetilde{E}_{i}$ is given by $-1^{M_{i}-1}$. By computing $A_{B_{*}, \tau} A_{B_{*}, \tau}^{-1}$, it is easily verified that $A_{B_{*}, \tau}^{-1}$ is indeed given by the foregoing expression. Now $A_{B_{*}, \tau}^{-1}$ is semi-lexicopositive if and only if $k_{i}$ is the largest integer such that $\left(i, k_{i}\right)$ is a best reply to $p$ for player $i$.

The next lemma shows that there are no $B_{*}$-complete facets $\tau$ in $\{0\} \times S$ where the cardinality of $B_{*}$ exceeds $N$.

Lemma 6.2. Let $(\Gamma, p, \Sigma)$ be given. Then there is no $B_{*}$-complete facet $\tau$ in $\{0\} \times S$ with $\left|B_{*}\right|>N$.

Proof. Consider any $\left|B_{*}\right|>N$ and any facet $\tau\left(\left(0, s^{1}\right), \ldots,\left(0, s^{m}\right)\right) \subset$ $\{0\} \times S\left(B_{*}\right)$ of a simplex in $\Sigma\left(B_{*}\right)$. Then

$$
A_{B_{*}, \tau}=\left[\begin{array}{ccccccccc}
1 & \cdots & 1 & 0 & \cdots & \cdots & \cdots & \cdots & 0 \\
& & & E_{1} & & 0 & -1^{M_{1}} & & 0 \\
\bar{G}\left(0, s^{1}\right) & \cdots & \bar{G}\left(0, s^{m}\right) & 0 & \ddots & 0 & 0 & \ddots & 0 \\
& & & 0 & 0 & E_{N} & 0 & 0 & -1^{M_{N}}
\end{array}\right] .
$$

Observe that $\bar{G}_{i k}\left(0, s^{j}\right)=R_{i}(p \backslash(i, k)),(i, k) \in \Phi_{i}$. So $\bar{G}\left(0, s^{j}\right)$ is independent of $j$ and $A_{B_{*}, \tau}$ is not invertible. Therefore, $\tau$ is certainly not $B_{*}$ complete. 
Lemma 6.1 selects for each player a unique pure strategy such that the resulting pure strategy combination, denoted by $s^{0}$, is a Nash equilibrium for the game $\Gamma^{0}$. Lemma 6.2 shows that there are no other Nash equilibria for the game $\Gamma^{0}$ that satisfy our criterion of completeness. The resulting point $\left(0, s^{0}\right)$ is linked in step 0 of Algorithm 4.5 to a point $\left(t, s^{1}\right)$ that is the other vertex of the unique simplex in $\Sigma\left(B_{*}^{0}\right)$ having $\{0\} \times S\left(B_{*}^{0}\right)$ as a facet.

It has already been indicated that Algorithm 4.5 generates a sequence of adjacent complete facets with varying dimension. The idea of adjacent is made precise in Definition 6.3.

Definition 6.3 (Adjacent Complete Facets). Let $(\Gamma, p, \Sigma)$ be given. The complete facets $\bar{\tau}$ and $\hat{\tau}$ are adjacent if $\bar{\tau}$ and $\hat{\tau}$ are both $B_{*}$-complete facets of the same simplex of $\Sigma\left(B_{*}\right)$, if $\bar{\tau}$ is a $\bar{B}_{*}$-complete facet of the $\widehat{B}_{*}$-complete simplex $\hat{\tau}$ of $\Sigma\left(\bar{B}_{*}\right)$, or if $\hat{\tau}$ is a $\widehat{B}_{*}$-complete facet of the $\bar{B}_{*}$-complete simplex $\bar{\tau}$ of $\Sigma\left(\widehat{B}_{*}\right)$.

THEOREM 6.4. Let $(\Gamma, p, \Sigma)$ be given. Consider a $B_{*}$-complete facet $\tau$. If $\tau=\{0\} \times S\left(B_{*}^{0}\right)$ or $\tau \subset\{1\} \times S$, then $\tau$ has one adjacent complete facet. Otherwise, $\tau$ has two adjacent complete facets.

Proof. Let $\tau=\{0\} \times S\left(B_{*}^{0}\right)$. Since $\{0\} \times S\left(B_{*}^{0}\right)$ is a subset of the relative boundary of $[0,1] \times S\left(B_{*}^{0}\right)$, there is a unique 1-simplex $\sigma \in \Sigma\left(B_{*}^{0}\right)$ such that $\tau$ is a facet of $\sigma$. By Lemma 4.3, it holds that either $\sigma$ has exactly one other $B_{*}^{0}$-complete facet $\bar{\tau}$ or the 1 -simplex $\sigma$ is $\bar{B}_{*}$-complete for precisely one admissible $\bar{B}_{*} \subset \Phi_{*}$. So there exists exactly one adjacent complete facet to $\{0\} \times S\left(B_{*}^{0}\right)$.

Let $\tau \subset\{1\} \times S$ be $B_{*}$ complete. Clearly, there is no admissible $\bar{B}_{*} \subset \Phi_{*}$ such that $\tau \in \Sigma\left(\bar{B}_{*}\right)$, since a simplex in $\Sigma\left(\bar{B}_{*}\right)$ always has vertices outside $\{1\} \times S$. Since $\tau$ is a subset of the relative boundary of $[0,1] \times S$, there is a unique 1-simplex $\sigma \in \Sigma\left(B_{*}\right)$ such that $\tau$ is a facet of $\sigma$. It follows again by Lemma 4.3 that there is exactly one adjacent complete facet to $\tau$.

For all other adjacent complete facets $\tau$, it holds that $\tau$ belongs either to the relative boundary of $[0,1] \times S$ or to the relative interior of $[0,1] \times S$. In the first case, there is a unique $\sigma \in \Sigma\left(B_{*}\right)$ having $\tau$ as a facet. By Lemma 4.3, it holds that either $\sigma$ has exactly one other $B_{*}$-complete facet $\bar{\tau}$ or that $\sigma$ is $\bar{B}_{*}$ complete for precisely one admissible $\bar{B}_{*} \subset \Phi_{*}$. This gives us one adjacent complete facet to $\tau$. Moreover, $\tau \in \Sigma\left(\overline{\bar{B}}_{*}\right)$ for precisely one admissible $\overline{\bar{B}}_{*} \subset \Phi_{*}$, since $\tau$ lies in the relative boundary of $\Sigma\left(B_{*}\right)$, $\tau \neq\{0\} \times S\left(B_{*}^{0}\right)$ by assumption, $\tau$ is not a subset of $\{1\} \times S$ by assumption, and $\tau \notin\{0\} \times S$ by Lemma 6.2. By Lemma 4.4, it holds that either $\tau$ is $\widehat{B}_{*}$ complete for precisely one admissible $\widehat{B}_{*} \subset \Phi_{*}$ with $\widehat{B}_{*} \neq B_{*}$ or precisely one facet $v$ of $\tau$ is $\overline{\bar{B}}_{*}$ complete. In the latter case, we are done. In the former case, since $\tau$ belongs to the relative boundary of $\Sigma\left(\widehat{B}_{*}\right)$, there is exactly one $\hat{\sigma} \in \Sigma\left(\widehat{B}_{*}\right)$ having $\tau$ as a facet, and applying Lemma 4.3 again 
gives the second adjacent complete facet to $\tau$. There can be no other adjacent complete facets to $\tau$.

When $\tau$ belongs to the relative interior of $\Sigma\left(B_{*}\right)$, we apply Lemma 4.3 twice to get exactly two adjacent complete facets.

The implications of Theorem 6.4 are striking. The $B_{*}^{0}$-complete facet $\{0\} \times S\left(B_{*}^{0}\right)$ has exactly one adjacent complete facet. That facet either belongs to the boundary of $\{1\} \times S$, and we have found an approximate Nash equilibrium, or has exactly one adjacent complete facet not equal to $\{0\} \times S\left(B_{*}^{0}\right)$. In this way, a sequence of adjacent complete facets is generated in a unique way, starting from $\{0\} \times S\left(B_{*}^{0}\right)$. The systematic steps of how to generate this sequence are given in Algorithm 4.5. Theorem 6.5 shows that the algorithm always reaches a complete facet in $\{1\} \times S$ in a finite number of steps. As stated in Theorem 6.6, this implies that the algorithm converges to an $\epsilon$-Nash equilibrium in a finite number of steps. It will usually not be the case that the boundary $\{1\} \times S$ is reached in one step. For this to occur, the triangulation $\Sigma$ must have a very large mesh size.

THEOREM 6.5 (Convergence of Algorithm 4.5). Let $(\Gamma, p, \Sigma)$ be given Then there exists a unique finite sequence of complete facets $\tau^{1}, \ldots, \tau^{\hat{k}}$ such that $\tau^{1}=\{0\} \times S\left(B_{*}^{0}\right), \tau^{\hat{k}} \subset\{1\} \times S$, and any two successive facets in the finite sequence are adjacent complete facets.

Proof. Let $\tau^{1}=\{0\} \times S\left(B_{*}^{0}\right)$. Let $\tau^{2}$ be the unique adjacent complete facet that exists according to Theorem 6.4. Whenever $\tau^{k}$ for some $k>1$ is not equal to $\{0\} \times S\left(B_{*}^{0}\right)$ and is not a subset of $\{1\} \times S$, there exists by Theorem 6.4 a unique adjacent complete facet $\tau^{k+1}$ not equal to $\tau^{k-1}$. Now it follows from the door-in door-out principle of Lemke and Howson (1964) that all simplices generated in the foregoing sequence are different. Moreover, the collection of all facets of simplices in $\Sigma\left(B_{*}\right)$ is finite for any admissible $B_{*} \subset \Phi_{*}$. So, after a finite number of steps, say $\hat{k}$, a facet in $\{1\} \times S$ must be reached.

By connecting the solutions found in each of the complete facets generated by the algorithm, we find the path generated by the algorithm, $\pi:[0,1] \rightarrow[0,1] \times S$, as constructed in Section 4 .

TheOREM 6.6. Let $\epsilon>0$ be given. Choose $\delta$ such that $\|\hat{x}-\tilde{x}\|_{\infty} \leq \delta$ implies $\|G(\hat{x})-G(\tilde{x})\|_{\infty} \leq \frac{1}{2} \epsilon, \hat{x}, \tilde{x} \in[0,1] \times S$. Let $(\Gamma, p, \Sigma)$ be such that mesh $(\Sigma) \leq \delta$. Then $\pi(r)=(t, s)$ implies that $s$ is an $\epsilon$-Nash equilibrium of $\Gamma^{t}$. Moreover, $\pi(0)=(0, s)$ with $s_{i k_{i}}=1$ for all $\left(i, k_{i}\right) \in B_{*}^{0}$, and $\pi(1)=\left(1, s^{*}\right)$ with $s^{*}$ an $\epsilon$-Nash equilibrium of $\Gamma$.

Proof. This follows straightforwardly from Theorem 3.2, Theorem 6.5, and the definition of Algorithm 4.5. 
Theorem 6.6 shows that any $(t, s)$ in the image set of $\pi$ corresponds to an $\epsilon$-Nash equilibrium of $\Gamma^{t}$. Since we have shown that the algorithm reaches a complete facet in $\{1\} \times S$ in a finite number of steps, we know that the algorithm converges to an $\epsilon$-Nash equilibrium in a finite number of steps. Notice that this result is true for any game and any prior, irrespective of possible degeneracies. It is clear that the inaccuracy of the final solution generated, $\epsilon$, depends on the mesh size of the triangulation. If the mesh size of the triangulation $\delta$ goes to 0 , then the inaccuracy $\epsilon$ can be chosen such as to go to 0 , since $G$ is a continuous function defined on a compact set. From a practical perspective, there is a clear trade-off between rapid convergence and very accurate solutions.

\section{CONVERGENCE TO THE LINEAR TRACING PROCEDURE}

It has been shown that Algorithm 4.5 converges to some $\epsilon$-Nash equilibrium, given any game and prior. It remains to be shown that this $\epsilon$-Nash equilibrium is indeed an approximation of the Nash equilibrium selected by the linear tracing procedure. To verify this, we need a distance function to measure the discrepancy between the set $\mathscr{L}^{\mathrm{c}}$ generated by the linear tracing procedure and points $\pi(r)$ which are generated by Algorithm 4.5.

Let $\mathscr{S}$ denote the collection of all nonempty compact subsets of $[0,1] \times S$. We define the distance function $d:[0,1] \times S \times \mathscr{S} \rightarrow \mathbb{R}$ by

$$
d(x, T)=\min _{y \in T}\|x-y\|_{\infty}, \quad x \in[0,1] \times S, \quad T \in \mathscr{S} .
$$

THEOREM 7.1. Let $(\Gamma, p)$ be given. Then for every $\epsilon>0$, there exists $\delta>0$ such that for every triangulation $\Sigma$ of $[0,1] \times S$ satisfying mesh $(\Sigma)<\delta$, it holds that $\max _{r \in[0,1]} d\left(\pi(r), \mathscr{L}^{\mathrm{c}}\right) \leq \epsilon$.

Proof. Suppose that the theorem is not true. Then there exists $\epsilon>0$ such that for every $n \in \mathbb{N}$, there exists a triangulation $\Sigma^{n}$ of $[0,1] \times S$ such that $\operatorname{mesh}\left(\Sigma^{n}\right)<\frac{1}{n}$, and there exists $r^{n} \in[0,1]$ such that $d\left(\pi^{n}\left(r^{n}\right), \mathscr{L}^{\mathrm{c}}\right)>\epsilon$. Here $\pi^{n}:[0,1] \rightarrow[0,1] \times S$ denotes the path generated by the algorithm when the triangulation $\Sigma^{n}$ is used. By Hildenbrand (1974, Proposition 1, p. 16), the sequence $\left\{\pi^{n}([0,1])\right\}_{n \in \mathbb{N}}$ has a convergent subsequence. By Theorem 3.2 and by continuity of the function $G$, it follows that the closed limit of a converging subsequence of $\left\{\pi^{n}([0,1])\right\}_{n \in \mathbb{N}}$ belongs to $\mathscr{L}$. Moreover, since $\pi^{n}([0,1])$ is connected for every $n \in \mathbb{N}$, the closed limit is connected by Mas-Colell (1985, Theorem A.5.1(ii), p. 10). But then the closed limit should be a subset of $\mathscr{L}^{\mathrm{c}}$, since it contains points in $\mathscr{L}^{0}$. Take any accumulation point $\bar{x}$ of the sequence $\left\{\pi^{n}\left(r^{n}\right)\right\}_{n \in \mathbb{N}}$. Then

$$
0=d\left(\bar{x}, \mathscr{L}^{\mathrm{c}}\right) \geq \inf \left\{d\left(\pi^{n}\left(r^{n}\right), \mathscr{L}^{\mathrm{c}}\right) \mid n \in \mathbb{N}\right\} \geq \epsilon,
$$

a contradiction. 
Theorem 7.1 shows that for all games, the algorithm stays arbitrarily close to the strategies defined by the linear tracing procedure. This holds true even if the linear tracing procedure is not well defined and does not select a unique Nash equilibrium. In that case, the $\epsilon$-Nash equilibrium generated by the algorithm is still an approximation of one of the Nash equilibria in $\mathscr{L}^{\mathrm{c}}$. Theorem 7.1 not only claims that $\pi(1)$ is close to a Nash equilibrium selected by the linear tracing procedure, but also makes clear that the entire path $\pi$ does not get further than $\epsilon$ away from $\mathscr{L}^{\mathrm{c}}$.

THEOREM 7.2. Let $(\Gamma, p)$ be given. If the linear tracing procedure is well defined, then for every $\epsilon>0$ there exists $\delta>0$ such that for every triangulation $\Sigma$ of $[0,1] \times S$ satisfying mesh $(\Sigma)<\delta$, it holds that $\max _{(t, s) \in \mathscr{L}^{c}} d((t, s)$, $\pi([0,1])) \leq \epsilon$. Moreover, if $\left(0, s^{0}\right) \in \mathscr{L}^{\mathrm{c}}$, then $d\left(\left(0, s^{0}\right),\{\pi(0)\}\right) \leq \epsilon$, and if $\left(1, s^{*}\right) \in \mathscr{L}^{\mathrm{c}}$, then $d\left(\left(1, s^{*}\right),\{\pi(1)\}\right) \leq \epsilon$.

Proof. Suppose that the theorem is not true. Then there exists $\epsilon>0$ such that for every $n \in \mathbb{N}$ there is a triangulation $\Sigma^{n}$ of $[0,1] \times S$ with $\operatorname{mesh}\left(\Sigma^{n}\right)<\frac{1}{n}$ and there is $\left(t^{n}, s^{n}\right) \in \mathscr{L}^{\mathrm{c}}$ such that $d\left(\left(t^{n}, s^{n}\right), \pi^{n}([0,1])\right)>$ $\epsilon$. Without loss of generality, the sequence $\left\{\left(t^{n}, s^{n}\right)\right\}_{n \in \mathbb{N}}$ is convergent, say to $(\bar{t}, \bar{s})$, and by Hildenbrand (1974, Proposition 1, p. 16), the sequence $\left\{\left(\pi^{n}([0,1])\right)\right\}_{n \in \mathbb{N}}$ is convergent, say to the set $\Pi$. By Theorem 7.1, it follows that $\Pi \subset \mathscr{L}^{\mathrm{c}}$. Obviously, $(\bar{t}, \bar{s}) \notin \Pi$. Since the set $\mathscr{L}^{0}$ is a polytope, well definedness of the linear tracing procedure implies that $\mathscr{L}^{0}$ contains a single point, say $\left(0, s^{0}\right)$. Similarly, well definedness yields that there is only one $(t, s) \in \mathscr{L}^{\mathrm{c}}$ such that $t=1$, say $\left(1, s^{1}\right)$. It follows that $\pi^{n}(0) \rightarrow\left(0, s^{0}\right)$ and $\pi^{n}(1) \rightarrow\left(1, s^{1}\right)$. The results of Schanuel et al. (1991) imply that $\mathscr{L}^{\mathrm{c}}$ is path connected. Then it is easily seen that the linear tracing procedure is well defined if and only if there is a homeomorphism $h:[0,1] \rightarrow \mathscr{L}^{\mathrm{c}}$ with $h(0)=\left(0, s^{0}\right)$ and $h(1)=\left(1, s^{1}\right)$. Moreover, there is $\bar{r} \in(0,1)$ such that $(\bar{t}, \bar{s})=h(\bar{r})$. By Mas-Colell (1985, Theorem A.5.1.(ii), p. 10), $\Pi$ is connected. However, $\Pi \subset h([0,1] \backslash\{\bar{r}\}), h(0) \in \Pi, h(1) \in \Pi$, and $h$ is a homeomorphism, and so $\Pi$ is not connected, a contradiction.

Theorem 7.2 claims that the path generated by the algorithm approximates every strategy of the linear tracing procedure if the linear tracing procedure is well defined. Obviously, if, for instance, the set $\mathscr{L}^{\mathrm{c}}$ has a branch point, then it cannot be expected that all points in $\mathscr{L}^{c}$ are approximated by the path $\pi$ generated by Algorithm 4.5. In this case the algorithm will track one of the branches that leads to an approximate Nash equilibrium.

Corollary 7.3. Let $(\Gamma, p)$ be given. Let $\left(\Sigma^{n}\right)_{n \in \mathbb{N}}$ be a sequence of triangulations of $[0,1] \times S$ with mesh size converging to 0 . If the linear tracing procedure is well defined, then $\pi^{n}([0,1])$ converges to $\mathscr{L}^{c}$ in the Hausdorff topology on $\mathscr{S}$. 
If the linear tracing procedure is well defined, then every strategy generated by it is approximated by Algorithm 4.5, (see Theorem 7.2), and every strategy generated by Algorithm 4.5 approximates a strategy corresponding to the linear tracing procedure according to Theorem 7.1. Since the linear tracing procedure is well defined for almost every game (see Harsanyi, 1975), this implies that the algorithm converges to the linear tracing procedure in the Hausdorff sense for almost all games.

\section{REFERENCES}

Doup, T. M., and Talman, A. J. J. (1987). "A Continuous Deformation Algorithm on the Product Space of Unit Simplices," Math. Oper. Res. 12, 485-521.

Eaves, B. C. (1971). "The Linear Complementarity Problem,” Manage. Sci. 17, 612-634.

Eaves, B. C. (1972). "Homotopies for Computation of Fixed Points," Math. Program. 3, 1-22.

Eaves, B. C., and Saigal, R. (1972). "Homotopies for Computation of Fixed Points on Unbounded Regions," Math. Program. 3, 225-237.

Garcia, C. B., Lemke, C. E., and Lüthi, H. J. (1973). "Simplicial Approximation of an Equilibrium Point of Noncooperative $N$-Person Games," in Mathematical Programming (T. C. Hu and S. M. Robinson, Eds.), pp. 227-260, New York: Academic Press.

Harsanyi, J. C. (1975). "The Tracing Procedure: A Bayesian Approach to Defining a Solution for $n$-Person Noncooperative Games," Int. J. Game Theory 4, 61-94.

Harsanyi, J. C., and Selten, R. (1988). A General Theory of Equilibrium Selection in Games. Cambridge, MA: MIT Press.

Herings, P. J. J., Talman, A. J. J., and Yang, Z. (1996). “The Computation of a Continuum of Constrained Equilibria," Math. Oper. Res. 21, 675-696.

Hildenbrand, W. (1974). Core and Equilibria of a Large Economy. Princeton, NJ: Princeton University Press.

Krohn, I., Moltzahn, S., Rosenmüller, J., Sudhölter, P. and Wallmeier, H. M. (1991). "Implementing the Modified LH Algorithm," Appl. Math. Comput. 45, 31-72.

Lemke, C. E., and Howson, J. T. Jr. (1964). "Equilibrium Points of Bimatrix Games," SIAM J. Appl. Math. 12, 413-423.

Mas-Colell, A. (1985). The Theory of General Economic Equilibrium, A Differentiable Approach. Cambridge, U.K.: Cambridge University Press.

Murty, K. G. (1983). Linear Programming. New York: Wiley.

Rosenmüller, J. (1971). "On a Generalization of the Lemke-Howson Algorithm to Noncooperative N-Person Games," SIAM J. Appl. Math. 21, 73-79.

Schanuel, S. H., Simon, L. K., and Zame, W. R. (1991). "The Algebraic Geometry of Games and the Tracing Procedure," in Game Equilibrium Models II: Methods, Morals and Markets (R. Selten, Ed.), pp. 9-43, Berlin: Springer-Verlag.

Todd, M. J. (1976a). The Computation of Fixed Points and Applications, Lecture Notes in Economics and Mathematical Systems 124. Berlin: Springer-Verlag.

Todd, M. J. (1976b). "On Triangulations for Computing Fixed Points," Math. Program. 10, 322-346. 
van Damme, E. (1999). "Game Theory: The Next Stage," in Economics Beyond the Millennium (A. P. Kirman and L. A. Gérard-Varet, Eds.), pp. 184-214. Oxford, U.K.: Oxford University Press.

van den Elzen, A. H. (1996). "Constructive Application of the Linear Tracing Procedure to Polymatrix Games," Research Memorandum FEW 738, Faculty of Economics, Tilburg University, Tilburg, The Netherlands.

van den Elzen, A. H., and Talman, A. J. J. (1991). "A Procedure for Finding Nash Equilibria in Bi-matrix Games," ZOR-Meth. Models Oper. Res. 35, 27-43.

van den Elzen, A. H., and Talman, A. J. J. (1999). "An Algorithmic Approach toward the Tracing Procedure for Bi-matrix Games," Games Econ. Behav. 28, 130-145.

van der Laan, G., and Talman, A. J. J. (1980). "A New Subdivision for Computing Fixed Points with a Homotopy Algorithm," Math. Program. 19, 78-91.

van der Laan, G., and Talman, A. J. J. (1982), "On the Computation of Fixed Points in the Product Space of Unit Simplices and an Application to Noncooperative N-Person Games," Math. Oper. Res. 7, 1-13.

Wilson, R. (1971). “Computing Equilibria of N-Person Games," SIAM J. Appl. Math. 21, 80-87. Wright, A. H. (1981). "The Octahedral Algorithm, a New Simplicial Fixed Point Algorithm," Math. Program. 21, 47-69. 